TRANSACTIONS OF THE

AMERICAN MATHEMATICAL SOCIETY

Volume 352, Number 6, Pages 2517-2552

S 0002-9947(00)02603-9

Article electronically published on February 29, 2000

\title{
ON MACAULAYFICATION OF NOETHERIAN SCHEMES
}

\author{
TAKESI KAWASAKI
}

\begin{abstract}
The Macaulayfication of a Noetherian scheme $X$ is a birational proper morphism from a Cohen-Macaulay scheme to $X$. In 1978 Faltings gave a Macaulayfication of a quasi-projective scheme if its non-Cohen-Macaulay locus is of dimension 0 or 1 . In the present article, we construct a Macaulayfication of Noetherian schemes without any assumption on the non-Cohen-Macaulay locus. Of course, a desingularization is a Macaulayfication and, in 1964, Hironaka already gave a desingularization of an algebraic variety over a field of characteristic 0. Our method, however, to construct a Macaulayfication is independent of the characteristic.
\end{abstract}

\section{INTRODUCTION}

Let $X$ be a Noetherian scheme. A birational proper morphism $Y \rightarrow X$ is said to be a Macaulayfication of $X$ if $Y$ is a Cohen-Macaulay scheme. This notion was given by Faltings 9] in analogy with desingularization. Furthermore, he proved that a quasi-projective scheme over a Noetherian ring $A$ has a Macaulayfication if its non-Cohen-Macaulay locus is of dimension 0 or 1 and $A$ possesses a dualizing complex. We should mention that his method of constructing a Macaulayfication is independent of the characteristic of the ring $A$ unlike Hironaka's desingularization [19.

Furthermore, several authors are interested in Macaulayfication. For example, Goto [10] gave a Macaulayfication of the spectrum of certain Buchsbaum rings. We can give a Macaulayfication of the spectrum of arbitrary Buchsbaum rings by Faltings' method. Goto's Macaulayfication, however, is better than Faltings' one in a sense. Indeed, Goto's one is a finite morphism though Faltings' one is not. Furthermore, Goto 11] and Schenzel [29, independently, refined Faltings' Macaulayfication in the case that the non-Cohen-Macaulay locus is of dimension 0.

On the other hand, Brodmann [4, 5] gave other Macaulayfications under the same assumption as Faltings. His method is quite different from Faltings' one. Indeed, his Macaulayfication preserves normality and regularity. He also showed in [6] that there exists no minimal Macaulayfication even if $X$ is a surface.

Recently, the author [21] gave a Macaulayfication of quasi-projective schemes whose non-Cohen-Macaulay locus is of dimension 2. In the present article, we improve it. The main theorem of this article is as follows.

Received by the editors November 11, 1996.

1991 Mathematics Subject Classification. Primary 14M05; Secondary 13H10, 14B05, 14E15.

Key words and phrases. Blowing-up, Cohen-Macaulay scheme, desingularization, dualizing complex, Macaulayfication.

The author is supported by Grant-in-Aid for Co-Operative Research. 
Theorem 1.1. Let $A$ be a Noetherian ring possessing a dualizing complex and $X$ a separated, of finite type scheme over $\operatorname{Spec} A$. Then $X$ has a Macaulayfication.

To make a survey, we sketch the construction of our Macaulayfication. First we consider the case of affine schemes. Let $A$ be a Noetherian local ring possessing a dualizing complex. Then the non-Cohen-Macaulay locus $V$ of $X=\operatorname{Spec} A$ is closed. We assume that $A$ is equidimensional and fix an integer $s$ such that $\operatorname{dim} V \leq s<$ $\operatorname{dim} X$. In this case our Macaulayfication of $X$ consists of successive $s+1$ blowingups

$$
Y_{s, 1} \rightarrow Y_{s, 2} \rightarrow \cdots \rightarrow Y_{s, s} \rightarrow Y_{s, s+1} \rightarrow X
$$

such that

$$
\operatorname{depth} \mathcal{O}_{Y_{s, i}, p} \geq d-i+1 \text { for all closed points } p \text { on } Y_{s, i} \text {. }
$$

The schemes $Y_{s, s}$ and $Y_{s, s+1}$ were essentially given by Faltings. Furthermore, if $s>0$, then we can give another Macaulayfication of $X$ consisting of a finite morphism $h_{s, 1}$ and successive $s$ blowing-ups

$$
Z_{s, 1} \stackrel{h_{s, 1}}{\longrightarrow} Y_{s, 2} \rightarrow \cdots \rightarrow Y_{s, s} \rightarrow Y_{s, s+1} \rightarrow X
$$

Since $s$ is arbitrary, we have distinct $2(\operatorname{dim} X-\operatorname{dim} V)$ Macaulayfications of $X$ if $\operatorname{dim} V>0$. In Section 4 we give the precise statement and its proof. We compute an example of a Macaulayfication in Appendix B

The center of each blowing-up is the ideal generated by a subsystem of certain system of parameters for $A$, named a $p$-standard system of parameters by Cuong [7]. In particular, the center of the first blowing-up is the ideal generated by an unconditioned strong $d$-sequence (for short $d^{+}$-sequence), which was studied by Goto and Yamagishi. We discuss a p-standard system of parameters in Sections 2 and 3 Since the theory of $d^{+}$-sequences plays a key role, this article includes proofs of a few results of [15] in Appendix A.

In Section $[5$ we prove Theorem 1.1 by using the result in the preceding section. It is a routine established by Faltings.

In Section [6] we give an application of Macaulayfication. A dualizing complex is an important notion for commutative algebra and algebraic geometry. It is wellknown that a homomorphic image of a finite-dimensional Gorenstein ring has a dualizing complex. Indeed if $B$ is a finite-dimensional Gorenstein ring, then its injective resolution $I^{\bullet}$ is a dualizing complex of $B$. If $A$ is a homomorphic image of $B$, then $\operatorname{Hom}_{B}\left(A, I^{\bullet}\right)$ is a dualizing complex of $A$. In 1979 Sharp [30] posed a conjecture: the converse is true. Aoyama and Goto [1], 2] gave a partial answer to Sharp's conjecture by using Faltings' Macaulayfication. They showed that Sharp's conjecture is true for a Noetherian local ring whose non-Cohen-Macaulay locus is of dimension 0 or 1 . Their argument still works in general. We show the following theorem.

Theorem 1.2. A Noetherian ring A possessing a dualizing complex is a homomorphic image of a finite-dimensional Gorenstein ring if A satisfies one of the following three conditions:

1. A has no embedded prime ideal and $t(\mathfrak{p})-\mathrm{ht} \mathfrak{p}$ is locally constant on $\operatorname{Spec} A$ where $t$ denotes the codimension function of $A$;

2. $A$ is an integral domain;

3. A is a local ring. 
Throughout this article except for Sections 5 and 6, $A$ denotes a Noetherian local ring with maximal ideal $\mathfrak{m}$. For a graded ring $R=\bigoplus_{n>0} R_{n}, R_{+}$denotes the irrelevant ideal $\bigoplus_{n>0} R_{n}$. We refer the reader to [17, [18] and [22] for other unexplained terminology.

\section{A P-STANDARD SYSTEM OF PARAMEters, I}

In this section, we state the definition and properties of a p-standard system of parameters, which was introduced by Cuong [7].

First we recall definitions of $d$-sequences and unconditioned strong $d$-sequences. They were given by Huneke [20] and GotoYamagishi [15], respectively, but our definition of $d$-sequences is slightly different from Huneke's original one.

Definition 2.1. Let $M$ be an $A$-module. A sequence $x_{1}, \ldots, x_{d}$ of elements in $A$ is said to be a $d$-sequence on $M$ if

$$
\left(x_{1}, \ldots, x_{i-1}\right) M: x_{i} x_{j}=\left(x_{1}, \ldots, x_{i-1}\right) M: x_{j} \quad \text { for any } 1 \leq i \leq j \leq d .
$$

A sequence $x_{1}, \ldots, x_{d}$ of elements in $A$ is said to be an unconditioned strong $d$ sequence (for short, a $d^{+}$-sequence) on $M$ if $x_{1}^{n_{1}}, \ldots, x_{d}^{n_{d}}$ is a $d$-sequence on $M$ for any positive integers $n_{1}, \ldots, n_{d}$ and in any order.

The following lemma was first given by Goto and Shimoda [12, Lemma 4.2] for the system of parameters for a Buchsbaum ring, which is a typical example of $d$-sequences. Since we often use it, we give a proof.

Lemma 2.2 ([15, Theorem 1.3]). Let $M$ be an A-module and $x_{1}, \ldots, x_{d}$ a $d$ sequence on $M$. If we put $\mathfrak{q}=\left(x_{1}, \ldots, x_{d}\right)$, then

$$
\left(x_{1}, \ldots, x_{i-1}\right) M: x_{i} \cap \mathfrak{q}^{n} M=\left(x_{1}, \ldots, x_{i-1}\right) \mathfrak{q}^{n-1} M
$$

for all $n>0$ and $1 \leq i \leq d+1$, where we set $x_{d+1}=1$ and $\left(x_{1}, \ldots, x_{i-1}\right)=(0)$ if $i=1$.

Proof. We work by induction on $n$ and $i$. If $i=d+1$, then it is trivial. Let $i \leq d$ and assume that

$$
\left(x_{1}, \ldots, x_{i}\right) M: x_{i+1} \cap \mathfrak{q}^{n} M=\left(x_{1}, \ldots, x_{i}\right) \mathfrak{q}^{n-1} M .
$$

If $a$ is in the left hand side of (2.2.1), then

$$
\begin{aligned}
a & \in\left(x_{1}, \ldots, x_{i-1}\right) M: x_{i} \cap \mathfrak{q}^{n} M \\
& \subseteq\left(x_{1}, \ldots, x_{i-1}\right) M: x_{i} x_{i+1} \cap \mathfrak{q}^{n} M \\
& =\left(x_{1}, \ldots, x_{i-1}\right) M: x_{i+1} \cap \mathfrak{q}^{n} M \\
& \subseteq\left(x_{1}, \ldots, x_{i}\right) M: x_{i+1} \cap \mathfrak{q}^{n} M \\
& =\left(x_{1}, \ldots, x_{i}\right) \mathfrak{q}^{n-1} M .
\end{aligned}
$$

Let $a=b+x_{i} c$ with $b \in\left(x_{1}, \ldots, x_{i-1}\right) \mathfrak{q}^{n-1} M$ and $c \in \mathfrak{q}^{n-1} M$. Then

$$
c \in\left(x_{1}, \ldots, x_{i-1}\right) M: x_{i}^{2}=\left(x_{1}, \ldots, x_{i-1}\right) M: x_{i} .
$$

If $n=1$, then $a=b+x_{i} c \in\left(x_{1}, \ldots, x_{i-1}\right) M$.

If $n>1$, then

$$
\begin{aligned}
c & \in\left(x_{1}, \ldots, x_{i-1}\right) M: x_{i} \cap \mathfrak{q}^{n-1} M \\
& =\left(x_{1}, \ldots, x_{i-1}\right) \mathfrak{q}^{n-2} M
\end{aligned}
$$

by the induction hypothesis. Thus $a=b+x_{i} c \in\left(x_{1}, \ldots, x_{i-1}\right) \mathfrak{q}^{n-1} M$. 
To state the definition of a p-standard system of parameters, we need the following definition and lemmas. They were given by Schenzel [26] [27] and [28].

Definition 2.3. Let $M$ be a finitely generated $A$-module. An ideal $\mathfrak{a}(M)$ is defined to be

$$
\mathfrak{a}(M)=\prod_{0 \leq i<\operatorname{dim} M} \operatorname{ann} H_{\mathfrak{m}}^{i}(M)
$$

where ann $H_{\mathfrak{m}}^{i}(M)$ denotes the annihilator of $H_{\mathfrak{m}}^{i}(M)$.

If $A$ is a homomorphic image of a Gorenstein local ring $B$, then the local duality theorem says that ann $H_{\mathfrak{m}}^{i}(M)=\operatorname{ann} \operatorname{Ext}_{B}^{n-i}(M, B)$, where $n=\operatorname{dim} B$. Therefore

$$
\mathfrak{a}(M)=\prod_{i=n-\operatorname{dim} M+1}^{n} \operatorname{ann} \operatorname{Ext}_{B}^{i}(M, B) .
$$

Of course, $M$ is Cohen-Macaulay if and only if $\mathfrak{a}(M)=A$.

Lemma 2.4. Let $M$ be a finitely generated A-module. If A possesses a dualizing complex, then the following statements hold:

1. $\operatorname{dim} A / \mathfrak{a}(M)<\operatorname{dim} M$.

2. Let $\mathfrak{p}$ be a prime ideal of $A$. Then $\mathfrak{a}(M) \nsubseteq \mathfrak{p}$ if and only if $M_{\mathfrak{p}}$ is a CohenMacaulay $A_{\mathfrak{p}}$-module and $\operatorname{dim} A / \mathfrak{p}+\operatorname{dim} M_{\mathfrak{p}}=\operatorname{dim} M$.

3. If $M$ is equidimensional, then the non-Cohen-Macaulay locus of $M$ coincides with $V(\mathfrak{a}(M))$.

Proof. (11): See [28, p. 46]. (2): See [28, Satz 2.4.6]. (3): See [28, p. 52].

Lemma 2.5. Let $M$ be a finitely generated A-module of dimension $d>0$ and $x_{1}, \ldots, x_{d}$ a system of parameters for $M$. Then

$$
\left(x_{1}, \ldots, x_{i-1}\right) M: x_{i} \subseteq\left(x_{1}, \ldots, x_{i-1}\right) M: \mathfrak{a}(M) \quad \text { for all } 1 \leq i \leq d .
$$

In particular, if $x_{i} \in \mathfrak{a}(M)$, then the equality holds.

Proof. See [27, Theorem 3] or [28. Satz 2.4.2].

Lemma 2.5 is always true but Lemma 2.4 is false without dualizing complex. We find a counterexample in [26].

Now we give the definition of a p-standard system of parameters, but it is slightly different from Cuong's original one.

Definition 2.6. Let $M$ be a finitely generated $A$-module of dimension $d>0$, $x_{1}, \ldots, x_{d}$ a system of parameters for $M$ and $s$ an integer such that $0 \leq s<d$. We say that $x_{1}, \ldots, x_{d}$ is a $p$-standard system of parameters of type $s$ if

1. $x_{s+1}, \ldots, x_{d} \in \mathfrak{a}(M)$;

2. $x_{i} \in \mathfrak{a}\left(M /\left(x_{i+1}, \ldots, x_{d}\right) M\right)$ for all $1 \leq i \leq s$.

In the case of $s=d-1$, our definition coincides with Cuong's one.

As a consequence of Lemma 2.4, we can find a p-standard system of parameters for any finitely generated module.

Theorem 2.7. Let $M$ be a finitely generated $A$-module of dimension $d>0$ and $s$ an integer such that $\operatorname{dim} A / \mathfrak{a}(M) \leq s<d$. If $A$ has a dualizing complex, then there exists a p-standard system of parameters of type $s$ for $M$. 
Proof. Since $d-\operatorname{dim} A / \mathfrak{a}(M) \geq d-s$, there exist $d-s$ elements $x_{s+1}, \ldots, x_{d} \in \mathfrak{a}(M)$ such that $\operatorname{dim} M /\left(x_{s+1}, \ldots, x_{d}\right) M=d-(d-s)=s$.

If a subsystem of parameters $x_{i+1}, \ldots, x_{d}$ for $M$ is given, then there exists $x_{i} \in \mathfrak{a}\left(M /\left(x_{s+1}, \ldots, x_{d}\right) M\right)$ such that $\operatorname{dim} M /\left(x_{i}, \ldots, x_{d}\right) M=i-1$, because $\operatorname{dim} A / \mathfrak{a}\left(M /\left(x_{i+1}, \ldots, x_{d}\right) M\right)<i$.

The following proposition comes from Lemma 2.5 at once.

Proposition 2.8. Let $M$ be a finitely generated A-module of dimension $d>0$ and $x_{1}, \ldots, x_{d}$ a p-standard system of parameters of type $s$ for $M$. Then the sequence $x_{s+1}, \ldots, x_{d}$ is a $d^{+}$-sequence on $M /\left(y_{1}, \ldots, y_{u}\right) M$ for any subsystem of parameters $y_{1}, \ldots, y_{u}$ for $M /\left(x_{s+1}, \ldots, x_{d}\right) M$. In particular, $x_{s+1}, \ldots, x_{d}$ is a $d^{+}$-sequence on $M$ itself.

Proof. Since $y_{1}, \ldots, y_{u}, x_{s+1}^{n_{s+1}}, \ldots, x_{i}^{n_{i}}$ and $y_{1}, \ldots, y_{u}, x_{s+1}^{n_{s+1}}, \ldots, x_{i-1}^{n_{i-1}}, x_{i}^{n_{i}} x_{j}^{n_{j}}$ are two subsystems of parameters for $M$,

$$
\begin{aligned}
\left(y_{1}, \ldots, y_{u}, x_{s+1}^{n_{s+1}}, \ldots, x_{i-1}^{n_{i-1}}\right) M: x_{i}^{n_{i}} & =\left(y_{1}, \ldots, y_{u}, x_{s+1}^{n_{s+1}}, \ldots, x_{i-1}^{n_{i-1}}\right) M: \mathfrak{a}(M) \\
& =\left(y_{1}, \ldots, y_{u}, x_{s+1}^{n_{s+1}}, \ldots, x_{i-1}^{n_{i-1}}\right) M: x_{i}^{n_{i}} x_{j}^{n_{j}}
\end{aligned}
$$

for any integers $n_{s+1}, \ldots, x_{d}$ and $s+1 \leq i \leq j \leq d$.

The main theorem of this section is as follows.

Theorem 2.9. Let $M$ be a finitely generated $A$-module of dimension $d>0, x_{1}, \ldots$, $x_{d}$ a p-standard system of parameters of type $s$ for $M$ and $y_{1}, \ldots, y_{u}$ a subsystem of parameters for $M /\left(x_{i}, \ldots, x_{d}\right) M$ where $2 \leq i \leq d$ and $1 \leq u<i$. If $y_{u} \in \mathfrak{a}(M)$ or $y_{u} \in \mathfrak{a}\left(M /\left(x_{i}, \ldots, x_{d}\right) M\right)$, then

$$
\left(y_{1}, \ldots, y_{v-1}, x_{\lambda} \mid \lambda \in \Lambda\right) M: y_{v} y_{u}=\left(y_{1}, \ldots, y_{v-1}, x_{\lambda} \mid \lambda \in \Lambda\right) M: y_{u}
$$

for any $1 \leq v \leq u$ and $\Lambda \subseteq\{i, \ldots, d\}$. In particular, by letting $\Lambda=\emptyset$, we have

$$
\left(y_{1}, \ldots, y_{v-1}\right) M: y_{v} y_{u}=\left(y_{1}, \ldots, y_{v-1}\right) M: y_{u} .
$$

Here $\left(x_{\lambda} \mid \lambda \in \Lambda\right)$ denotes the ideal generated by $\left\{x_{\lambda} \mid \lambda \in \Lambda\right\}$ and we put

$$
\left(y_{1}, \ldots, y_{v-1}, x_{\lambda} \mid \lambda \in \Lambda\right)=\left(y_{1}, \ldots, y_{v-1}\right)+\left(x_{\lambda} \mid \lambda \in \Lambda\right) .
$$

Proof. If $y_{u} \in \mathfrak{a}(M)$, then the both side of (2.9.1) coincide with

$$
\left(y_{1}, \ldots, y_{v-1}, x_{\lambda} \mid \lambda \in \Lambda\right) M: \mathfrak{a}(M)
$$

because of Lemma 2.5

In the case of $y_{u} \in \mathfrak{a}\left(M /\left(x_{i}, \ldots, x_{d}\right) M\right)$, we work by induction on the number of elements in $\Lambda$. If $\Lambda=\{i, \ldots, d\}$, then (2.9.1) comes from Lemma 2.5 applied to $M /\left(x_{i}, \ldots, x_{d}\right) M$.

Assume that $\Lambda \neq\{i, \ldots, d\}$ and let $l$ be the largest element of $\{i, \ldots, d\} \backslash \Lambda$. Let $a$ be an element of the left hand side of (2.9.1). Then we have

$$
\begin{aligned}
a & \in\left(y_{1}, \ldots, y_{v-1}, x_{l}, x_{\lambda} \mid \lambda \in \Lambda\right) M: y_{v} y_{u} \\
& =\left(y_{1}, \ldots, y_{v-1}, x_{l}, x_{\lambda} \mid \lambda \in \Lambda\right) M: y_{u}
\end{aligned}
$$

by the induction hypothesis. We put $y_{u} a=x_{l} b+c$ with

$$
c \in\left(y_{1}, \ldots, y_{v-1}, x_{\lambda} \mid \lambda \in \Lambda\right) M .
$$


Then we obtain that

$$
\begin{aligned}
b & \in\left(y_{1}, \ldots, y_{v-1}, x_{\lambda} \mid \lambda \in \Lambda\right) M: y_{v} x_{l} \\
& =\left(y_{1}, \ldots, y_{v-1}, x_{\lambda} \mid \lambda \in \Lambda\right) M: x_{l} .
\end{aligned}
$$

Indeed, if $l \leq s$, then $x_{l} \in \mathfrak{a}\left(M /\left(x_{l+1}, \ldots, x_{d}\right) M\right)$ and $l+1, \ldots, d \in \Lambda$. Hence we can apply Lemma 2.5 to $M /\left(x_{l+1}, \ldots, x_{d}\right) M$. On the other hand, if $l>s$, then $x_{l} \in \mathfrak{a}(M)$. We can apply Lemma 2.5 to $M$ itself. Therefore

$$
y_{u} a=x_{l} b+c \in\left(y_{1}, \ldots, y_{v-1}, x_{\lambda} \mid \lambda \in \Lambda\right) M
$$

That is, $a \in\left(y_{1}, \ldots, y_{v-1}, x_{\lambda} \mid \lambda \in \Lambda\right) M: y_{u}$. The proof is completed.

We use the following corollary for our Macaulayfication in Section 4.

Corollary 2.10. Let $M$ be a finitely generated $A$-module of dimension $d>0$, $x_{1}, \ldots, x_{d}$ a p-standard system of parameters of type $s$ for $M$ and $y_{1}, \ldots, y_{u} a$ subsystem of parameters for $M /\left(x_{i}, \ldots, x_{d}\right) M$ where $1 \leq i \leq d$ and $1 \leq u<i$. Then the sequence $x_{i}, \ldots, x_{d}$ is a d-sequence on $M /\left(y_{1}, \ldots, y_{u}\right) M$. In particular, $x_{1}, \ldots, x_{d}$ is a d-sequence on $M$ itself.

Proof. Let $i \leq j \leq k \leq d$. By applying (2.9.2) to the subsystem of parameters $y_{1}, \ldots, y_{u}, x_{i}, \ldots, x_{k}$ for $M /\left(x_{k+1}, \ldots, x_{d}\right) M$, we obtain

$$
\left(y_{1}, \ldots, y_{u}, x_{i}, \ldots, x_{j-1}\right) M: x_{j} x_{k}=\left(y_{1}, \ldots, y_{u}, x_{i}, \ldots, x_{j-1}\right) M: x_{k} \text {. }
$$

\section{A P-STANDARD SYSTEM OF PARAMETERS, II}

This section is devoted to the proof of the following theorem.

Theorem 3.1. Let $M$ be a finitely generated $A$-module of dimension $d>0$ and $x_{1}, \ldots, x_{d}$ a p-standard system of parameters of type $s$ for $M$. We put $\mathfrak{q}_{i}=$ $\left(x_{i}, \ldots, x_{d}\right)$ for all $1 \leq i \leq d$. Then for any positive integers $1 \leq i \leq j \leq d$ and $n_{i}, \ldots, n_{j}$, we obtain the following statements:

$\left(A_{i j}\right)$ If $y_{1}, \ldots, y_{u}$ is a subsystem of parameters for $M / \mathfrak{q}_{i} M$, then

$$
\begin{aligned}
\left(y_{1}, \ldots, y_{u}, x_{k}, \ldots, x_{l-1}\right) M: x_{l} \cap\left[\left(y_{1}, \ldots, y_{u}\right) M+\mathfrak{q}_{i}^{n_{i}} \cdots \mathfrak{q}_{j}^{n_{j}} M\right] \\
=\left(y_{1}, \ldots, y_{u}\right) M+\left(x_{k}, \ldots, x_{l-1}\right) \mathfrak{q}_{i}^{n_{i}} \cdots \mathfrak{q}_{k}^{n_{k}-1} \cdots \mathfrak{q}_{j}^{n_{j}} M
\end{aligned}
$$

for arbitrary integers $k$ and $l$ such that $i \leq k \leq j$ and $k \leq l \leq d+1$. Here we set $x_{d+1}=1$.

$\left(B_{i j}\right)$ If $y_{1}, \ldots, y_{u}$ is a subsystem of parameters for $M / \mathfrak{q}_{i} M$, then

$$
\begin{aligned}
& {\left[\left(y_{1}, \ldots, y_{u-1}\right) M+\left(x_{k}, \ldots, x_{l}\right) \mathfrak{q}_{i}^{n_{i}} \cdots \mathfrak{q}_{j}^{n_{j}} M\right]: y_{u}} \\
& \quad=\left(x_{k}, \ldots, x_{l}\right)\left\{\left[\left(y_{1}, \ldots, y_{u-1}\right) M+\mathfrak{q}_{i}^{n_{i}} \cdots \mathfrak{q}_{j}^{n_{j}} M\right]: y_{u}\right\} \\
& \quad+\left(y_{1}, \ldots, y_{u-1}\right) M: y_{u}
\end{aligned}
$$

for arbitrary integers $k$ and $l$ such that $i \leq k \leq j$ and $k \leq l \leq d$. In particular, by letting $l=d$, we have

$$
\begin{aligned}
{\left[\left(y_{1}, \ldots, y_{u-1}\right) M+\right.} & \left.\mathfrak{q}_{i}^{n_{i}} \cdots \mathfrak{q}_{k}^{n_{k}+1} \cdots \mathfrak{q}_{j}^{n_{j}} M\right]: y_{u} \\
& =\mathfrak{q}_{k}\left\{\left[\left(y_{1}, \ldots, y_{u-1}\right) M+\mathfrak{q}_{i}^{n_{i}} \cdots \mathfrak{q}_{j}^{n_{j}} M\right]: y_{u}\right\} \\
& +\left(y_{1}, \ldots, y_{u-1}\right) M: y_{u}
\end{aligned}
$$

for all $i \leq k \leq j$. 
$\left(C_{i j}\right)$ If $y_{1}, \ldots, y_{u}$ is a subsystem of parameters for $M / \mathfrak{q}_{i} M$, then

$$
\begin{aligned}
{\left[\left(y_{1}, \ldots, y_{u-1}\right) M+\mathfrak{q}_{i}^{n_{i}} \cdots \mathfrak{q}_{j}^{n_{j}} M\right] } & : y_{u} \\
& \subseteq\left(y_{1}, \ldots, y_{u-1}\right) M: y_{u}+\mathfrak{q}_{i}^{n_{i}-1} \cdots \mathfrak{q}_{j}^{n_{j}} M .
\end{aligned}
$$

$\left(D_{i j}\right)$ If $y_{1}, \ldots, y_{u}$ is a subsystem of parameters for $M / \mathfrak{q}_{i} M$, then

$$
\begin{aligned}
& {\left[\left(y_{1}, \ldots, y_{u-1}\right) M+\mathfrak{q}_{i} \cdots \mathfrak{q}_{j} M\right]: y_{u} \cap x_{i} M} \\
& \quad \subseteq x_{i}\left\{\left[\left(y_{1}, \ldots, y_{u-1}\right) M+\mathfrak{q}_{i+1} \cdots \mathfrak{q}_{j} M\right]: y_{u}\right\}+\left(y_{1}, \ldots, y_{u-1}\right) M .
\end{aligned}
$$

Here we put $\mathfrak{q}_{i+1} \cdots \mathfrak{q}_{j} M=M$ if $i=j$.

$\left(E_{i j}\right)$ Let $k$ be an integer such that $2 \leq k \leq i$. If $y_{1}, \ldots, y_{u}$ is a subsystem of parameters for $M / \mathfrak{q}_{k} M$ such that $y_{u} \in \mathfrak{a}\left(M / \mathfrak{q}_{k} M\right)$, then

$$
\begin{aligned}
{\left[\left(y_{1}, \ldots, y_{v-1}, x_{\lambda} \mid \lambda\right.\right.} & \left.\in \Lambda) M+\mathfrak{q}_{i}^{n_{i}} \cdots \mathfrak{q}_{j}^{n_{j}} M\right]: y_{v} y_{u} \\
& =\left[\left(y_{1}, \ldots, y_{v-1}, x_{\lambda} \mid \lambda \in \Lambda\right) M+\mathfrak{q}_{i}^{n_{i}} \cdots \mathfrak{q}_{j}^{n_{j}} M\right]: y_{u}
\end{aligned}
$$

for any $1 \leq v \leq u$ and $\Lambda \subseteq\{k, \ldots, i-1\}$.

We divide the proof into several steps. Roughly speaking, we work by induction on $k=j-i$.

\section{DIAGRAM OF THE INDUCTION}

$\begin{array}{lll}\forall i:\left(A_{i i}\right),\left(B_{i i}\right), \ldots,\left(E_{i, i+k-1}\right) & \Rightarrow \forall i:\left(A_{i, i+k}\right) & \text { (Steps 1 and 5); } \\ \forall i:\left(A_{i i}\right),\left(B_{i i}\right), \ldots,\left(E_{i, i+k-1}\right),\left(A_{i, i+k}\right) & \Rightarrow \forall i:\left(B_{i, i+k}\right) & \text { (Step 2); } \\ \forall i:\left(A_{i i}\right),\left(B_{i i}\right), \ldots,\left(A_{i, i+k}\right),\left(B_{i, i+k}\right) & \Rightarrow \forall i:\left(C_{i, i+k}\right) & \text { (Steps 3 and 6); } \\ \forall i:\left(A_{i i}\right),\left(B_{i i}\right), \ldots,\left(B_{i, i+k}\right),\left(C_{i, i+k}\right) & \Rightarrow \forall i:\left(D_{i, i+k}\right) & \text { (Steps [1 and 7); } \\ \forall i:\left(A_{i i}\right),\left(B_{i i}\right), \ldots,\left(C_{i, i+k}\right),\left(D_{i, i+k}\right) & \Rightarrow \forall i:\left(E_{i, i+k}\right) & \text { (Steps 3, 8 and 9). }\end{array}$

Step 1. $\left(A_{i i}\right)$ is true.

Proof. Corollary [2.10] says that $x_{i}, \ldots, x_{d}$ is a $d$-sequence on $M /\left(y_{1}, \ldots, y_{u}\right) M$. Therefore $\left(A_{i i}\right)$ coincides with Lemma 2.2

Step 2. If $j \geq i$, then $\left(B_{i j}\right)$ comes from $\left(A_{i j}\right)$.

Proof. Let $a$ be an element of the left hand side of 3.1.2 and put $y_{u} a=x_{l} b+c$ with $b \in \mathfrak{q}_{i}^{n_{i}} \cdots \mathfrak{q}_{j}^{n_{j}} M$ and $c \in\left(y_{1}, \ldots, y_{u-1}\right) M+\left(x_{k}, \ldots, x_{l-1}\right) \mathfrak{q}_{i}^{n_{i}} \cdots \mathfrak{q}_{j}^{n_{j}} M$. By using $\left(A_{i j}\right)$, we obtain

$$
\begin{aligned}
b & \in\left(y_{1}, \ldots, y_{u}, x_{k}, \ldots, x_{l-1}\right) M: x_{l} \cap \mathfrak{q}_{i}^{n_{i}} \cdots \mathfrak{q}_{j}^{n_{j}} M \\
& \subseteq\left(y_{1}, \ldots, y_{u}\right) M+\left(x_{k}, \ldots, x_{l-1}\right) \mathfrak{q}_{i}^{n_{i}} \cdots \mathfrak{q}_{k}^{n_{k}-1} \cdots \mathfrak{q}_{j}^{n_{j}} M .
\end{aligned}
$$

Let $b=y_{u} a^{\prime}+c^{\prime}$ with

$$
c^{\prime} \in\left(y_{1}, \ldots, y_{u-1}\right) M+\left(x_{k}, \ldots, x_{l-1}\right) \mathfrak{q}_{i}^{n_{i}} \cdots \mathfrak{q}_{k}^{n_{k}-1} \cdots \mathfrak{q}_{j}^{n_{j}} M
$$

Then

$$
a^{\prime} \in\left[\left(y_{1}, \ldots, y_{u-1}\right) M+\mathfrak{q}_{i}^{n_{i}} \cdots \mathfrak{q}_{j}^{n_{j}} M\right]: y_{u}
$$

and

$$
a-x_{l} a^{\prime} \in\left[\left(y_{1}, \ldots, y_{u-1}\right) M+\left(x_{k}, \ldots, x_{l-1}\right) \mathfrak{q}_{i}^{n_{i}} \cdots \mathfrak{q}_{j}^{n_{j}} M\right]: y_{u}
$$


By induction on $l$, we find that $a$ is in the right hand side of (3.1.2). The opposite inclusion is obvious.

Step 3. $\left(B_{i i}\right)$ implies $\left(C_{i i}\right)$ and $\left(E_{i i}\right)$.

Proof. By using $\left(B_{i i}\right)$, we have

$$
\begin{aligned}
{\left[\left(y_{1}, \ldots, y_{u-1}\right) M+\right.} & \left.\mathfrak{q}_{i}^{n_{i}} M\right]: y_{u} \\
= & \mathfrak{q}_{i}^{n_{i}-1}\left\{\left[\left(y_{1}, \ldots, y_{u-1}\right) M+\mathfrak{q}_{i} M\right]:\right. \\
& \left.y_{u}\right\} \\
& +\left(y_{1}, \ldots, y_{u-1}\right) M: y_{u}
\end{aligned}
$$

and

$$
\begin{aligned}
{\left[\left(y_{1}, \ldots, y_{v-1}, x_{\lambda} \mid \lambda \in \Lambda\right) M+\mathfrak{q}_{i}^{n_{i}} M\right]: y_{v} y_{u} } & \\
=\mathfrak{q}_{i}^{n_{i}-1}\left\{\left[\left(y_{1}, \ldots, y_{v-1}, x_{\lambda} \mid \lambda \in \Lambda\right) M+\right.\right. & \left.\left.\mathfrak{q}_{i} M\right]: y_{v} y_{u}\right\} \\
& +\left(y_{1}, \ldots, y_{v-1}\right) M: y_{v} y_{u} .
\end{aligned}
$$

Furthermore, if $y_{u} \in \mathfrak{a}\left(M / \mathfrak{q}_{k} M\right)$, then (2.9.2) says that

$$
\left(y_{1}, \ldots, y_{v-1}\right) M: y_{v} y_{u}=\left(y_{1}, \ldots, y_{v-1}\right) M: y_{u} .
$$

Thus we may assume that $n_{i}=1$. Then $\left(C_{i i}\right)$ is trivial and $\left(E_{i i}\right)$ is included in Theorem 2.9.

Step 4. $\left(D_{i i}\right)$ is trivial.

Proof. Indeed, the right hand side of (3.1.4) is $\left(y_{1}, \ldots, y_{u-1}, x_{i}\right) M$.

Step 5. If $j>i$, then $\left(A_{i j}\right)$ comes from $\left(A_{i+1, j}\right)$ and $\left(C_{i+1, j}\right)$.

Proof. We first show that

$$
\begin{array}{r}
\left(y_{1}, \ldots, y_{u}, x_{i}, \ldots, x_{l-1}\right) M: x_{l} \cap\left[\left(y_{1}, \ldots, y_{u-1}\right) M+\mathfrak{q}_{i+1}^{n_{i+1}} \cdots \mathfrak{q}_{j}^{n_{j}} M\right] \\
\subseteq\left(y_{1}, \ldots, y_{u}\right) M+\left(x_{i}, \ldots, x_{l-1}\right) \mathfrak{q}_{i+1}^{n_{i+1}-1} \cdots \mathfrak{q}_{j}^{n_{j}} M
\end{array}
$$

for all $i \leq l \leq d+1$. If $l=d+1$, then (3.1.6) is trivial. Assume that $l \leq d$. Let $a$ be an element of the left hand side of (3.1.6). By applying $\left(A_{i+1, j}\right)$ to a subsystem of parameters $y_{1}, \ldots, y_{u}, x_{i}$ for $M / \mathfrak{q}_{i+1} M$, we obtain

$$
\begin{aligned}
a & \in\left(y_{1}, \ldots, y_{u}, x_{i}, \ldots, x_{l-1}\right) M: x_{l} \cap\left[\left(y_{1}, \ldots, y_{u}, x_{i}\right) M+\mathfrak{q}_{i+1}^{n_{i+1}} \cdots \mathfrak{q}_{j}^{n_{j}} M\right] \\
& =\left(y_{1}, \ldots, y_{u}, x_{i}\right) M+\left(x_{i+1}, \ldots, x_{l-1}\right) \mathfrak{q}_{i+1}^{n_{i+1}-1} \cdots \mathfrak{q}_{j}^{n_{j}} M .
\end{aligned}
$$

If we put $a=x_{i} b+c$ with

$$
c \in\left(y_{1}, \ldots, y_{u}\right) M+\left(x_{i+1}, \ldots, x_{l-1}\right) \mathfrak{q}_{i+1}^{n_{i+1}-1} \cdots \mathfrak{q}_{j}^{n_{j}} M
$$

then

$$
\begin{aligned}
b & \in\left[\left(y_{1}, \ldots, y_{u}\right) M+\mathfrak{q}_{i+1}^{n_{i+1}} \cdots \mathfrak{q}_{j}^{n_{j}} M\right]: x_{i} \\
& \subseteq\left(y_{1}, \ldots, y_{u}\right) M: x_{i}+\mathfrak{q}_{i+1}^{n_{i+1}-1} \cdots \mathfrak{q}_{j}^{n_{j}} M
\end{aligned}
$$

by $\left(C_{i+1, j}\right)$ applied to a subsystem of parameters $y_{1}, \ldots, y_{u}, x_{i}$ for $M / \mathfrak{q}_{i+1} M$. If $l=i$, then the left hand side of (3.1.6) is contained in

$$
\left(y_{1}, \ldots, y_{u}\right) M: x_{i} \cap\left(y_{1}, \ldots, y_{u}, x_{i}, \ldots, x_{d}\right) M=\left(y_{1}, \ldots, y_{u}\right) M .
$$

Therefore $a$ is in the right hand side. 
Next we show (3.1.1). In the case of $k=i$, we work by induction on $l$ and $n_{i}$. Let $a$ be an element of the left hand side of (3.1.1). If $l=d+1$, then there is nothing to prove. Assume that $i+1 \leq l \leq d$. Since $x_{i}, \ldots, x_{d}$ is a $d$-sequence on $M /\left(y_{1}, \ldots, y_{u}\right) M$,

$$
\left(y_{1}, \ldots, y_{u}, x_{i}, \ldots, x_{l-1}\right) M: x_{l} \subseteq\left(y_{1}, \ldots, y_{u}, x_{i}, \ldots, x_{l}\right) M: x_{l+1}
$$

as the proof of Lemma 2.2. Hence we have

$$
\begin{aligned}
a & \in\left(y_{1}, \ldots, y_{u}, x_{i}, \ldots, x_{l}\right) M: x_{l+1} \cap\left[\left(y_{1}, \ldots, y_{u}\right) M+\mathfrak{q}_{i}^{n_{i}} \cdots \mathfrak{q}_{j}^{n_{j}} M\right] \\
& =\left(y_{1}, \ldots, y_{u}\right) M+\left(x_{i}, \ldots, x_{l}\right) \mathfrak{q}_{i}^{n_{i}-1} \cdots \mathfrak{q}_{j}^{n_{j}} M
\end{aligned}
$$

by the induction hypothesis. Let $a=x_{l} a^{\prime}+b$ with $a^{\prime} \in \mathfrak{q}_{i}^{n_{i}-1} \cdots \mathfrak{q}_{j}^{n_{j}} M$ and

$$
b \in\left(y_{1}, \ldots, y_{u}\right) M+\left(x_{i}, \ldots, x_{l-1}\right) \mathfrak{q}_{i}^{n_{i}-1} \cdots \mathfrak{q}_{j}^{n_{j}} M .
$$

Since $x_{i}, \ldots, x_{d}$ is a $d$-sequence on $M /\left(y_{1}, \ldots, y_{u}\right) M$,

$$
a^{\prime} \in\left(y_{1}, \ldots, y_{u}, x_{i}, \ldots, x_{l-1}\right) M: x_{l}^{2}=\left(y_{1}, \ldots, y_{u}, x_{i}, \ldots, x_{l-1}\right) M: x_{l} .
$$

Therefore we get

$$
\begin{aligned}
a^{\prime} & \in\left(y_{1}, \ldots, y_{u}, x_{i}, \ldots, x_{l-1}\right) M: x_{l} \cap \mathfrak{q}_{i}^{n_{i}-1} \ldots \mathfrak{q}_{j}^{n_{j}} M \\
& \subseteq \begin{cases}\left(y_{1}, \ldots, y_{u}\right) M+\left(x_{i}, \ldots, x_{l-1}\right) \mathfrak{q}_{i+1}^{n_{i+1}-1} \ldots \mathfrak{q}_{j}^{n_{j}} M & \text { if } n_{i}=1 \\
\left(y_{1}, \ldots, y_{u}\right) M+\left(x_{i}, \ldots, x_{l-1}\right) \mathfrak{q}_{i}^{n_{i}-2} \ldots \mathfrak{q}_{j}^{n_{j}} M & \text { if } n_{i}>1\end{cases}
\end{aligned}
$$

by using (3.1.6) or the induction hypothesis, respectively. Since $x_{l} \in \mathfrak{q}_{i+1} \subseteq \mathfrak{q}_{i}$, $a=x_{l} a^{\prime}+b$ is in the right hand side of (3.1.1). If $l=i$, then

$$
\begin{aligned}
a & \in\left(y_{1}, \ldots, y_{u}\right) M: x_{i} \cap\left[\left(y_{1}, \ldots, y_{u}\right) M+\mathfrak{q}_{i}^{n_{i}} \cdots \mathfrak{q}_{j}^{n_{j}} M\right] \\
& \subseteq\left(y_{1}, \ldots, y_{u}\right) M: x_{i} \cap\left(y_{1}, \ldots, y_{u}, x_{i}, \ldots, x_{d}\right) M \\
& =\left(y_{1}, \ldots, y_{u}\right) M
\end{aligned}
$$

because of Lemma 2.2. The proof is completed if $k=i$.

In the case of $k>i$, we work by induction on $n_{i}$. Assume that $l \leq d$. Let $a$ be an element of the left hand side of (3.1.1). Since $\left(x_{i}\right)+\mathfrak{q}_{i}^{n_{i}}=\left(x_{i}\right)+\mathfrak{q}_{i+1}^{n_{i}}$, we have

$$
\begin{aligned}
a & \in\left(y_{1}, \ldots, y_{u}, x_{i}, x_{k}, \ldots, x_{l-1}\right) M: x_{l} \cap\left[\left(y_{1}, \ldots, y_{u}, x_{i}\right) M+\mathfrak{q}_{i+1}^{n_{i}+n_{i+1}} \cdots \mathfrak{q}_{j}^{n_{j}} M\right] \\
& = \begin{cases}\left(y_{1}, \ldots, y_{u}, x_{i}\right) M+\left(x_{i+1}, \ldots, x_{l-1}\right) \mathfrak{q}_{i+1}^{n_{i}+n_{i+1}-1} \cdots \mathfrak{q}_{j}^{n_{j}} M & \text { if } k=i+1 ; \\
\left(y_{1}, \ldots, y_{u}, x_{i}\right) M+\left(x_{k}, \ldots, x_{l-1}\right) \mathfrak{q}_{i+1}^{n_{i}+n_{i+1}} \cdots \mathfrak{q}_{k}^{n_{k}-1} \cdots \mathfrak{q}_{j}^{n_{j}} M & \text { if } k>i+1\end{cases} \\
& =\left(y_{1}, \ldots, y_{u}, x_{i}\right) M+\left(x_{k}, \ldots, x_{l-1}\right) \mathfrak{q}_{i}^{n_{i}} \cdots \mathfrak{q}_{k}^{n_{k}-1} \cdots \mathfrak{q}_{j}^{n_{j}} M .
\end{aligned}
$$

Here we applied $\left(A_{i+1, j}\right)$ to the subsystem of parameters $y_{1}, \ldots, y_{u}, x_{i}$ for $M / \mathfrak{q}_{i+1} M$. Taking intersection with $\left(y_{1}, \ldots, y_{u}\right) M+\mathfrak{q}_{i}^{n_{i}} \cdots \mathfrak{q}_{j}^{n_{j}} M$,

$$
\begin{aligned}
a \in & \left(y_{1}, \ldots, y_{u}, x_{i}\right) M \cap\left[\left(y_{1}, \ldots, y_{u}\right) M+\mathfrak{q}_{i}^{n_{i}} \cdots \mathfrak{q}_{j}^{n_{j}} M\right] \\
& +\left(x_{k}, \ldots, x_{l-1}\right) \mathfrak{q}_{i}^{n_{i}} \cdots \mathfrak{q}_{k}^{n_{k}-1} \cdots \mathfrak{q}_{j}^{n_{j}} M \\
= & \left(y_{1}, \ldots, y_{u}\right) M+x_{i} \mathfrak{q}_{i}^{n_{i}-1} \cdots \mathfrak{q}_{j}^{n_{j}} M \\
& +\left(x_{k}, \ldots, x_{l-1}\right) \mathfrak{q}_{i}^{n_{i}} \cdots \mathfrak{q}_{k}^{n_{k}-1} \cdots \mathfrak{q}_{j}^{n_{j}} M .
\end{aligned}
$$

Here we used (3.1.1) in the case of $k=i$ to show the equation. Let $a=x_{i} a^{\prime}+b$ with $a^{\prime} \in \mathfrak{q}_{i}^{n_{i}-1} \cdots \mathfrak{q}_{j}^{n_{j}} M$ and

$$
b \in\left(y_{1}, \ldots, y_{u}\right) M+\left(x_{k}, \ldots, x_{l-1}\right) \mathfrak{q}_{i}^{n_{i}} \cdots \mathfrak{q}_{k}^{n_{k}-1} \cdots \mathfrak{q}_{j}^{n_{j}} M
$$


By applying (2.9.2) to the subsystem of parameters $y_{1}, \ldots, y_{u}, x_{k}, \ldots, x_{l-1}, x_{i}$, $x_{l}$ for $M / \mathfrak{q}_{l+1} M$, we obtain

$$
a^{\prime} \in\left(y_{1}, \ldots, y_{u}, x_{k}, \ldots, x_{l-1}\right) M: x_{i} x_{l}=\left(y_{1}, \ldots, y_{u}, x_{k}, \ldots, x_{l-1}\right) M: x_{l} .
$$

Therefore we have

$$
\begin{aligned}
a^{\prime} & \in\left(y_{1}, \ldots, y_{u}, x_{k}, \ldots, x_{l-1}\right) M: x_{l} \cap \mathfrak{q}_{i}^{n_{i}-1} \cdots \mathfrak{q}_{j}^{n_{j}} M \\
& \subseteq \begin{cases}\left(y_{1}, \ldots, y_{u}\right) M+\left(x_{k}, \ldots, x_{l-1}\right) \mathfrak{q}_{i+1}^{n_{i+1}} \cdots \mathfrak{q}_{k}^{n_{k}-1} \cdots \mathfrak{q}_{j}^{n_{j}} M & \text { if } n_{i}=1 ; \\
\left(y_{1}, \ldots, y_{u}\right) M+\left(x_{k}, \ldots, x_{l-1}\right) \mathfrak{q}_{i}^{n_{i}-1} \cdots \mathfrak{q}_{k}^{n_{k}-1} \cdots \mathfrak{q}_{j}^{n_{j}} M & \text { if } n_{i}>1\end{cases}
\end{aligned}
$$

by $\left(A_{i+1, j}\right)$ or the induction hypothesis, respectively. Therefore $a=x_{i} a^{\prime}+b$ is in the right hand side of (3.1.1). The opposite inclusion is obvious.

Step 6. If $j>i$, then $\left(C_{i j}\right)$ comes from $\left(B_{i j}\right),\left(C_{i i}\right),\left(C_{i+1, j}\right)$ and $\left(E_{i+1, j}\right)$.

Proof. We first show that

$$
\left(y_{1}, \ldots, y_{u-1}, x_{i}\right) M: y_{u} \cap\left(y_{1}, \ldots, y_{u-1}, x_{i}, \ldots, x_{l}\right) M=\left(y_{1}, \ldots, y_{u-1}, x_{i}\right) M
$$

for all $i \leq l \leq d$. We work by induction on $l$. If $l=i$, then there is nothing to prove. Assume that $l>i$ and let $a$ be an element of the left hand side of (3.1.7). We put $a=x_{l} b+c$ with $c \in\left(y_{1}, \ldots, y_{u-1}, x_{i}, \ldots, x_{l-1}\right) M$. By applying (2.9.2) to a subsystem of parameters $y_{1}, \ldots, y_{u-1}, x_{i}, \ldots, x_{l-1}, y_{u}, x_{l}$ for $M / \mathfrak{q}_{l+1} M$, we have

$$
\begin{aligned}
b & \in\left(y_{1}, \ldots, y_{u-1}, x_{i}, \ldots, x_{l-1}\right) M: y_{u} x_{l} \\
& =\left(y_{1}, \ldots, y_{u-1}, x_{i}, \ldots, x_{l-1}\right) M: x_{l} .
\end{aligned}
$$

Thus we get

$$
\begin{aligned}
a & \in\left(y_{1}, \ldots, y_{u-1}, x_{i}\right) M: y_{u} \cap\left(y_{1}, \ldots, y_{u-1}, x_{i}, \ldots, x_{l-1}\right) M \\
& =\left(y_{1}, \ldots, y_{u-1}, x_{i}\right) M
\end{aligned}
$$

by the induction hypothesis. The opposite inclusion is obvious.

Next we show (3.1.3). In the same way as Step [3 we may assume that $n_{i}=$ $\cdots=n_{j}=1$. Let $a$ be an element of the left hand side of (3.1.3). By $\left(C_{i+1, j}\right)$ we obtain

$$
\begin{aligned}
a & \in\left[\left(y_{1}, \ldots, y_{u-1}, x_{i}\right) M+\mathfrak{q}_{i+1}^{2} \cdots \mathfrak{q}_{j} M\right]: y_{u} \\
& \subseteq\left(y_{1}, \ldots, y_{u-1}, x_{i}\right) M: y_{u}+\mathfrak{q}_{i+1} \cdots \mathfrak{q}_{j} M .
\end{aligned}
$$

On the other hand, $\left(C_{i i}\right)$ gives

$$
\begin{aligned}
a & \in\left[\left(y_{1}, \ldots, y_{u-1}\right) M+\mathfrak{q}_{i}^{2} M\right]: y_{u} \\
& \subseteq\left(y_{1}, \ldots, y_{u-1}\right) M: y_{u}+\mathfrak{q}_{i} M .
\end{aligned}
$$

Thus

$$
\begin{aligned}
a & \in\left[\left(y_{1}, \ldots, y_{u-1}, x_{i}\right) M: y_{u}+\mathfrak{q}_{i+1} \cdots \mathfrak{q}_{j} M\right] \cap\left[\left(y_{1}, \ldots, y_{u-1}\right) M: y_{u}+\mathfrak{q}_{i} M\right] \\
& =\left(y_{1}, \ldots, y_{u-1}\right) M: y_{u}+\mathfrak{q}_{i+1} \cdots \mathfrak{q}_{j} M+\left(y_{1}, \ldots, y_{u-1}, x_{i}\right) M: y_{u} \cap \mathfrak{q}_{i} M \\
& =\left(y_{1}, \ldots, y_{u-1}\right) M: y_{u}+\mathfrak{q}_{i+1} \cdots \mathfrak{q}_{j} M+x_{i} M .
\end{aligned}
$$

Here we used (3.1.7) to show the last equation. Taking intersection with

$$
\left[\left(y_{1}, \ldots, y_{u-1}\right) M+\mathfrak{q}_{i+1} \cdots \mathfrak{q}_{j} M\right]: y_{u},
$$


we have

$$
\begin{aligned}
a \in & \left(y_{1}, \ldots, y_{u-1}\right) M: y_{u}+\mathfrak{q}_{i+1} \cdots \mathfrak{q}_{j} M \\
& +\left[\left(y_{1}, \ldots, y_{u-1}\right) M+\mathfrak{q}_{i+1} \cdots \mathfrak{q}_{j} M\right]: y_{u} \cap x_{i} M \\
= & \left(y_{1}, \ldots, y_{u-1}\right) M: y_{u}+\mathfrak{q}_{i+1} \cdots \mathfrak{q}_{j} M \\
& +x_{i}\left\{\left[\left(y_{1}, \ldots, y_{u-1}\right) M+\mathfrak{q}_{i+1} \cdots \mathfrak{q}_{j} M\right]: y_{u} x_{i}\right\} .
\end{aligned}
$$

By applying $\left(E_{i+1, j}\right)$ to a subsystem of parameters $y_{1}, \ldots, y_{u}, x_{i}$ for $M / \mathfrak{q}_{i+1} M$, we have

$$
\left[\left(y_{1}, \ldots, y_{u-1}\right) M+\mathfrak{q}_{i+1} \cdots \mathfrak{q}_{j} M\right]: y_{u} x_{i}=\left[\left(y_{1}, \ldots, y_{u-1}\right) M+\mathfrak{q}_{i+1} \cdots \mathfrak{q}_{j} M\right]: x_{i} .
$$

Therefore $a \in\left(y_{1}, \ldots, y_{u-1}\right) M: y_{u}+\mathfrak{q}_{i+1} \cdots \mathfrak{q}_{j} M$. The proof is completed.

Step 7. If $j>i$, then $\left(C_{i+1, j}\right)$ implies $\left(D_{i j}\right)$.

Proof. We first show that

$$
\begin{aligned}
& {\left[\left(y_{1}, \ldots, y_{u-1}\right) M+x_{i} \mathfrak{q}_{i+1} \cdots \mathfrak{q}_{j} M\right]: y_{u}} \\
& =x_{i}\left\{\left[\left(y_{1}, \ldots, y_{u-1}\right) M+\mathfrak{q}_{i+1} \cdots \mathfrak{q}_{j} M\right]: y_{u}\right\} \\
& \quad+\left(y_{1}, \ldots, y_{u-1}\right) M: y_{u} .
\end{aligned}
$$

Let $a$ be an element of the left hand side of (3.1.8) and put $y_{u} a=x_{i} b+c$ with $b \in \mathfrak{q}_{i+1} \cdots \mathfrak{q}_{j} M$ and $c \in\left(y_{1}, \ldots, y_{u-1}\right) M$. Then

$$
\begin{aligned}
b & \in\left(y_{1}, \ldots, y_{u}\right) M: x_{i} \cap \mathfrak{q}_{i} M \\
& \subseteq\left(y_{1}, \ldots, y_{u}\right) M
\end{aligned}
$$

because $x_{i}, \ldots, x_{d}$ is a $d$-sequence on $M /\left(y_{1}, \ldots, y_{u}\right) M$. We put $b=y_{u} a^{\prime}+c^{\prime}$ with $c^{\prime} \in\left(y_{1}, \ldots, y_{u-1}\right) M$. Then $a^{\prime} \in\left[\left(y_{1}, \ldots, y_{u-1}\right) M+\mathfrak{q}_{i+1} \cdots \mathfrak{q}_{j} M\right]: y_{u}$ and $a-x_{i} a^{\prime} \in\left(y_{1}, \ldots, y_{u-1}\right) M: y_{u}$. Hence $a$ is in the right hand side of (3.1.8) and the opposite inclusion is obvious.

Next we show (3.1.4). Let $a$ be an element of $M$ such that

$$
x_{i} a \in\left[\left(y_{1}, \ldots, y_{u-1}\right) M+\mathfrak{q}_{i} \cdots \mathfrak{q}_{j} M\right]: y_{u}
$$

and put $y_{u} x_{i} a=x_{i} b+b^{\prime}$ with $b \in \mathfrak{q}_{i+1} \cdots \mathfrak{q}_{j} M$ and

$$
b^{\prime} \in\left(y_{1}, \ldots, y_{u-1}\right) M+\mathfrak{q}_{i+1}^{2} \cdots \mathfrak{q}_{j} M .
$$

By applying $\left(C_{i+1, j}\right)$ to a subsystem of parameters $y_{1}, \ldots, y_{u-1}, x_{i}$ for $M / \mathfrak{q}_{i+1} M$, we have

$$
\begin{aligned}
y_{u} a-b & \in\left[\left(y_{1}, \ldots, y_{u-1}\right) M+\mathfrak{q}_{i+1}^{2} \cdots \mathfrak{q}_{j} M\right]: x_{i} \\
& \subseteq\left(y_{1}, \ldots, y_{u-1}\right) M: x_{i}+\mathfrak{q}_{i+1} \cdots \mathfrak{q}_{j} M
\end{aligned}
$$

and hence

$$
y_{u} a \in\left(y_{1}, \ldots, y_{u-1}\right) M: x_{i}+\mathfrak{q}_{i+1} \cdots \mathfrak{q}_{j} M
$$

Therefore

$$
\begin{aligned}
x_{i} a \in & {\left[\left(y_{1}, \ldots, y_{u-1}\right) M+x_{i} \mathfrak{q}_{i+1} \cdots \mathfrak{q}_{j} M\right]: y_{u} \cap x_{i} M } \\
= & x_{i}\left\{\left[\left(y_{1}, \ldots, y_{u-1}\right) M+\mathfrak{q}_{i+1} \cdots \mathfrak{q}_{j} M\right]: y_{u}\right\} \\
& +\left(y_{1}, \ldots, y_{u-1}\right) M: y_{u} \cap x_{i} M .
\end{aligned}
$$


Here we used (3.1.8). By applying (2.9.2) to a subsystem of parameters $y_{1}, \ldots$, $y_{u}, x_{i}$ for $M / \mathfrak{q}_{i+1} M$, we have

$$
\left(y_{1}, \ldots, y_{u-1}\right) M: y_{u} x_{i}=\left(y_{1}, \ldots, y_{u-1}\right) M: x_{i} .
$$

Hence we have

$$
\begin{aligned}
\left(y_{1}, \ldots, y_{u-1}\right) M: y_{u} \cap x_{i} M & =x_{i}\left[\left(y_{1}, \ldots, y_{u-1}\right) M: y_{u} x_{i}\right] \\
& =x_{i}\left[\left(y_{1}, \ldots, y_{u-1}\right) M: x_{i}\right] \\
& \subseteq\left(y_{1}, \ldots, y_{u-1}\right) M .
\end{aligned}
$$

Hence $x_{i} a$ is in the right hand side of (3.1.4). Thus the proof is completed.

Step 8. $\left(E_{i, i+1}\right)$ comes from $\left(B_{i, i+1}\right),\left(E_{i i}\right)$ and $\left(E_{i+1, i+1}\right)$.

Proof. In the same way as Step 3, we may assume that $n_{i}=n_{i+1}=1$. Let $a$ be an element of the left hand side of 3.1.5. Then we have, by $\left(E_{i i}\right)$,

$$
\begin{aligned}
a & \in\left[\left(y_{1}, \ldots, y_{v-1}, x_{\lambda} \mid \lambda \in \Lambda\right) M+\mathfrak{q}_{i}^{2} M\right]: y_{v} y_{u} \\
& =\left[\left(y_{1}, \ldots, y_{v-1}, x_{\lambda} \mid \lambda \in \Lambda\right) M+\mathfrak{q}_{i}^{2} M\right]: y_{u} .
\end{aligned}
$$

Therefore we put $y_{u} a=x_{i}^{2} b+c$ with $c \in\left(y_{1}, \ldots, y_{v-1}, x_{\lambda} \mid \lambda \in \Lambda\right) M+\mathfrak{q}_{i} \mathfrak{q}_{i+1} M$. On the other hand, $\left(E_{i+1, i+1}\right)$ says that

$$
\begin{aligned}
a & \in\left[\left(y_{1}, \ldots, y_{v-1}, x_{\lambda} \mid \lambda \in \Lambda\right) M+\mathfrak{q}_{i+1} M\right]: y_{v} y_{u} \\
& \subseteq\left[\left(y_{1}, \ldots, y_{v-1}, x_{\lambda} \mid \lambda \in \Lambda\right) M+\mathfrak{q}_{i+1} M\right]: y_{u} .
\end{aligned}
$$

Thus

$$
\begin{aligned}
b & \in\left[\left(y_{1}, \ldots, y_{v-1}, x_{\lambda} \mid \lambda \in \Lambda\right) M+\mathfrak{q}_{i+1} M\right]: x_{i}^{2} \\
& =\left[\left(y_{1}, \ldots, y_{v-1}, x_{\lambda} \mid \lambda \in \Lambda\right) M+\mathfrak{q}_{i+1} M\right]: x_{i} .
\end{aligned}
$$

Indeed, $x_{i} \in \mathfrak{a}(M)$ or $x_{i} \in \mathfrak{a}\left(M / \mathfrak{q}_{i+1} M\right)$. Therefore

$$
y_{u} a=x_{i}^{2} b+c \in\left(y_{1}, \ldots, y_{v-1}, x_{\lambda} \mid \lambda \in \Lambda\right) M+\mathfrak{q}_{i} \mathfrak{q}_{i+1} M .
$$

The proof is completed.

Step 9. If $j>i+1$, then $\left(E_{i j}\right)$ is followed from $\left(A_{i j}\right),\left(B_{i j}\right),\left(C_{i+1, j}\right),\left(D_{i j}\right)$ and $\left(E_{i+1, j}\right)$.

Proof. In the same way as Step B we may assume that $n_{i}=\cdots=n_{j}=1$. Let $a$ be an element of the left hand side of (3.1.5) $)$. By applying $\left(E_{i+1, j}\right)$, we have

$$
\begin{aligned}
a & \in\left[\left(y_{1}, \ldots, y_{v-1}, x_{i}, x_{\lambda} \mid \lambda \in \Lambda\right) M+\mathfrak{q}_{i+1}^{2} \cdots \mathfrak{q}_{j} M\right]: y_{v} y_{u} \\
& =\left[\left(y_{1}, \ldots, y_{v-1}, x_{i}, x_{\lambda} \mid \lambda \in \Lambda\right) M+\mathfrak{q}_{i+1}^{2} \cdots \mathfrak{q}_{j} M\right]: y_{u} .
\end{aligned}
$$

Therefore

$$
\begin{aligned}
y_{u} a \in & {\left[\left(y_{1}, \ldots, y_{v-1}, x_{\lambda} \mid \lambda \in \Lambda\right) M+\mathfrak{q}_{i} \cdots \mathfrak{q}_{j} M\right]: y_{v} } \\
& \cap\left[\left(y_{1}, \ldots, y_{v-1}, x_{i}, x_{\lambda} \mid \lambda \in \Lambda\right) M+\mathfrak{q}_{i+1}^{2} \cdots \mathfrak{q}_{j} M\right] \\
= & \left(y_{1}, \ldots, y_{v-1}, x_{\lambda} \mid \lambda \in \Lambda\right) M+\mathfrak{q}_{i+1}^{2} \cdots \mathfrak{q}_{j} M \\
& +\left[\left(y_{1}, \ldots, y_{v-1}, x_{\lambda} \mid \lambda \in \Lambda\right) M+\mathfrak{q}_{i} \cdots \mathfrak{q}_{j} M\right]: y_{v} \cap x_{i} M \\
= & \left(y_{1}, \ldots, y_{v-1}, x_{\lambda} \mid \lambda \in \Lambda\right) M+\mathfrak{q}_{i+1}^{2} \cdots \mathfrak{q}_{j} M \\
& +x_{i}\left\{\left[\left(y_{1}, \ldots, y_{v-1}, x_{\lambda} \mid \lambda \in \Lambda\right) M+\mathfrak{q}_{i+1} \cdots \mathfrak{q}_{j} M\right]: y_{v}\right\} .
\end{aligned}
$$


Here we used $\left(D_{i j}\right)$ to show the second equation. We put $y_{u} a=x_{i} b+c$ with

$$
b \in\left[\left(y_{1}, \ldots, y_{v-1}, x_{\lambda} \mid \lambda \in \Lambda\right) M+\mathfrak{q}_{i+1} \cdots \mathfrak{q}_{j} M\right]: y_{v}
$$

and

$$
c \in\left(y_{1}, \ldots, y_{v-1}, x_{\lambda} \mid \lambda \in \Lambda\right) M+\mathfrak{q}_{i+1}^{2} \cdots \mathfrak{q}_{j} M .
$$

By applying $\left(C_{i+1, j}\right)$ to a subsystem of parameters $y_{1}, \ldots, y_{v-1}, y_{u},\left\{x_{\lambda} \mid \lambda \in \Lambda\right\}$, $x_{i}$ for $M / \mathfrak{q}_{i+1} M$, we obtain

$$
\begin{aligned}
b & \in\left[\left(y_{1}, \ldots, y_{v-1}, y_{u}, x_{\lambda} \mid \lambda \in \Lambda\right) M+\mathfrak{q}_{i+1}^{2} \cdots \mathfrak{q}_{j} M\right]: x_{i} \\
& \subseteq\left(y_{1}, \ldots, y_{v-1}, y_{u}, x_{\lambda} \mid \lambda \in \Lambda\right) M: x_{i}+\mathfrak{q}_{i+1} \cdots \mathfrak{q}_{j} M .
\end{aligned}
$$

Therefore

$$
\begin{aligned}
b \in & {\left[\left(y_{1}, \ldots, y_{v-1}, x_{\lambda} \mid \lambda \in \Lambda\right) M+\mathfrak{q}_{i+1} \cdots \mathfrak{q}_{j} M\right]: y_{v} } \\
& \cap\left[\left(y_{1}, \ldots, y_{v-1}, y_{u}, x_{\lambda} \mid \lambda \in \Lambda\right) M: x_{i}+\mathfrak{q}_{i+1} \cdots \mathfrak{q}_{j} M\right] \\
= & {\left[\left(y_{1}, \ldots, y_{v-1}, x_{\lambda} \mid \lambda \in \Lambda\right) M+\mathfrak{q}_{i+1} \cdots \mathfrak{q}_{j} M\right]: y_{v} } \\
& \cap\left(y_{1}, \ldots, y_{v-1}, y_{u}, x_{\lambda} \mid \lambda \in \Lambda\right) M: x_{i} \\
& +\mathfrak{q}_{i+1} \cdots \mathfrak{q}_{j} M .
\end{aligned}
$$

Since $j \geq i+2$, we have

$$
\begin{aligned}
{\left[\left(y_{1}, \ldots, y_{v-1}, x_{\lambda} \mid \lambda \in \Lambda\right) M+\mathfrak{q}_{i+1}\right.} & \left.\cdots \mathfrak{q}_{j} M\right]: y_{v} \\
& \subseteq\left(y_{1}, \ldots, y_{v-1}, x_{\lambda} \mid \lambda \in \Lambda\right) M: y_{v}+\mathfrak{q}_{i+2} M
\end{aligned}
$$

by using $\left(C_{i+1, j}\right)$. Furthermore, we obtain

$$
\begin{aligned}
\left(y_{1}, \ldots, y_{v-1}, x_{\lambda} \mid \lambda \in \Lambda\right) M: y_{v} & \subseteq\left(y_{1}, \ldots, y_{v-1}, x_{\lambda} \mid \lambda \in \Lambda\right) M: y_{v} x_{i} \\
& =\left(y_{1}, \ldots, y_{v-1}, x_{\lambda} \mid \lambda \in \Lambda\right) M: x_{i} \\
& \subseteq\left(y_{1}, \ldots, y_{v-1}, y_{u}, x_{\lambda} \mid \lambda \in \Lambda\right) M: x_{i}
\end{aligned}
$$

by using (2.9.2). Therefore

$$
\begin{aligned}
\left(y_{1}, \ldots, y_{v-1}, y_{u}, x_{\lambda} \mid \lambda \in \Lambda\right) M: x_{i} \cap & {\left[\left(y_{1}, \ldots, y_{v-1}, x_{\lambda} \mid \lambda \in \Lambda\right) M: y_{v}+\mathfrak{q}_{i+2} M\right] } \\
= & \left(y_{1}, \ldots, y_{v-1}, x_{\lambda} \mid \lambda \in \Lambda\right) M: y_{v} \\
& +\left(y_{1}, \ldots, y_{v-1}, y_{u}, x_{\lambda} \mid \lambda \in \Lambda\right) M: x_{i} \cap \mathfrak{q}_{i+2} M \\
\subseteq & \left(y_{1}, \ldots, y_{v-1}, x_{\lambda} \mid \lambda \in \Lambda\right) M: y_{v}+y_{u} M .
\end{aligned}
$$

Here we applied Lemma 2.2 to a $d$-sequence $x_{i}, \ldots, x_{d}$ on

$$
M /\left(y_{1}, \ldots, y_{v-1}, y_{u}, x_{\lambda} \mid \lambda \in \Lambda\right) M .
$$


Thus

$$
\begin{aligned}
b \in & {\left[\left(y_{1}, \ldots, y_{v-1}, x_{\lambda} \mid \lambda \in \Lambda\right) M+\mathfrak{q}_{i+1} \cdots \mathfrak{q}_{j} M\right]: y_{v} } \\
& \cap\left[\left(y_{1}, \ldots, y_{v-1}, x_{\lambda} \mid \lambda \in \Lambda\right) M: y_{v}+\mathfrak{q}_{i+2} M\right] \\
& \cap\left(y_{1}, \ldots, y_{v-1}, y_{u}, x_{\lambda} \mid \lambda \in \Lambda\right) M: x_{i} \\
& +\mathfrak{q}_{i+1} \cdots \mathfrak{q}_{j} M \\
\subseteq & {\left[\left(y_{1}, \ldots, y_{v-1}, x_{\lambda} \mid \lambda \in \Lambda\right) M+\mathfrak{q}_{i+1} \cdots \mathfrak{q}_{j} M\right]: y_{v} } \\
& \cap\left[\left(y_{1}, \ldots, y_{v-1}, x_{\lambda} \mid \lambda \in \Lambda\right) M: y_{v}+y_{u} M\right] \\
& +\mathfrak{q}_{i+1} \cdots \mathfrak{q}_{j} M \\
= & {\left[\left(y_{1}, \ldots, y_{v-1}, x_{\lambda} \mid \lambda \in \Lambda\right) M+\mathfrak{q}_{i+1} \cdots \mathfrak{q}_{j} M\right]: y_{v} \cap y_{u} M } \\
& +\left(y_{1}, \ldots, y_{v-1}, x_{\lambda} \mid \lambda \in \Lambda\right) M: y_{v} \\
& +\mathfrak{q}_{i+1} \ldots \mathfrak{q}_{j} M \\
= & y_{u}\left\{\left[\left(y_{1}, \ldots, y_{v-1}, x_{\lambda} \mid \lambda \in \Lambda\right) M+\mathfrak{q}_{i+1} \cdots \mathfrak{q}_{j} M\right]: y_{v} y_{u}\right\} \\
& +\left(y_{1}, \ldots, y_{v-1}, x_{\lambda} \mid \lambda \in \Lambda\right) M: y_{v} \\
& +\mathfrak{q}_{i+1} \cdots \mathfrak{q}_{j} M \\
= & \left(y_{1}, \ldots, y_{v-1}, x_{\lambda} \mid \lambda \in \Lambda\right) M: y_{v}+\mathfrak{q}_{i+1} \cdots \mathfrak{q}_{j} M .
\end{aligned}
$$

Here we used $\left(E_{i+1, j}\right)$ to show the last equation. Thus we have

$$
y_{u} a=x_{i} b+c \in\left(y_{1}, \ldots, y_{v-1}, x_{\lambda} \mid \lambda \in \Lambda\right) M+\mathfrak{q}_{i} \cdots \mathfrak{q}_{j} M,
$$

where we used (3.1.9) again. The opposite inclusion is obvious.

Thus we finish the proof of Theorem 3.1

The following corollary immediately comes from Theorem 3.1. We, however, can easily prove it if $j=i+1$. See [21, Lemma 5.4].

Corollary 3.2. With the same notation as Theorem [3.1, we have

$$
\left[\left(y_{1}, \ldots, y_{u}\right) M+\mathfrak{q}_{i}^{n_{i}} \cdots \mathfrak{q}_{j}^{n_{j}} M\right]: x_{i-1}^{n_{i-1}}=\left[\left(y_{1}, \ldots, y_{u}\right) M+\mathfrak{q}_{i}^{n_{i}} \cdots \mathfrak{q}_{j}^{n_{j}} M\right]: \mathfrak{q}_{i-1}
$$

for any positive integers $2 \leq i \leq j \leq d, n_{i-1}, \ldots, n_{j}$ and a subsystem of parameters $y_{1}, \ldots, y_{u}$ for $M / \mathfrak{q}_{i-1} M$.

Proof. We may assume that $n_{i-1}=1$ by using $\left(E_{i j}\right)$. Then we have

$$
\left[\left(y_{1}, \ldots, y_{u}\right) M+\mathfrak{q}_{i}^{n_{i}} \cdots \mathfrak{q}_{j}^{n_{j}} M\right]: x_{i-1} \subseteq\left(y_{1}, \ldots, y_{u}\right) M: x_{i-1}+\mathfrak{q}_{i}^{n_{i}-1} \cdots \mathfrak{q}_{j}^{n_{j}} M
$$

by applying $\left(C_{i j}\right)$ to a subsystem of parameters $y_{1}, \ldots, y_{u}, x_{i-1}$ for $M / \mathfrak{q}_{i} M$. Since $x_{i-1}, \ldots, x_{d}$ is a $d$-sequence on $M /\left(y_{1}, \ldots, y_{u}\right) M$,

$$
\left(y_{1}, \ldots, y_{u}\right) M: x_{i-1} \subseteq\left(y_{1}, \ldots, y_{u}\right) M: \mathfrak{q}_{i-1} .
$$

Therefore

$$
\mathfrak{q}_{i-1}\left\{\left[\left(y_{1}, \ldots, y_{u}\right) M+\mathfrak{q}_{i}^{n_{i}} \cdots \mathfrak{q}_{j}^{n_{j}} M\right]: x_{i-1}\right\} \subseteq\left(y_{1}, \ldots, y_{u}\right) M+\mathfrak{q}_{i}^{n_{i}} \cdots \mathfrak{q}_{j}^{n_{j}} M .
$$

The opposite inclusion is obvious.

We also need the following corollary for our Macaulayfication.

Corollary 3.3. With the same notation as Theorem 3.1, we let $k$ be an integer such that $1 \leq k \leq d$ and $y_{1}, \ldots, y_{u}$ a subsystem of parameters for $M / \mathfrak{q}_{k} M$. If

$$
\left[\left(y_{1}, \ldots, y_{u-1}\right) M+\mathfrak{q}_{k} M\right]: y_{u}=\left(y_{1}, \ldots, y_{u-1}\right) M+\mathfrak{q}_{k} M,
$$


then

$$
\left(y_{1}, \ldots, y_{u-1}\right) M: y_{u}=\left(y_{1}, \ldots, y_{u-1}\right) M
$$

and

$$
\begin{aligned}
{\left[\left(y_{1}, \ldots, y_{u-1}, x_{\lambda} \mid \lambda \in \Lambda\right) M\right.} & \left.+\mathfrak{q}_{i}^{n_{i}} \cdots \mathfrak{q}_{j}^{n_{j}} M\right]: y_{u} \\
& =\left(y_{1}, \ldots, y_{u-1}, x_{\lambda} \mid \lambda \in \Lambda\right) M+\mathfrak{q}_{i}^{n_{i}} \cdots \mathfrak{q}_{j}^{n_{j}} M
\end{aligned}
$$

for any positive integers $k \leq i \leq j, n_{i}, \ldots, n_{j}$ and $\Lambda \subseteq\{k, \ldots, i-1\}$.

Proof. First we show that

$$
\left(y_{1}, \ldots, y_{u-1}, x_{\lambda} \mid \lambda \in \Lambda\right) M: y_{u}=\left(y_{1}, \ldots, y_{u-1}, x_{\lambda} \mid \lambda \in \Lambda\right) M
$$

for any $\Lambda \subseteq\{k, \ldots, d\}$. We work by descending induction on the number of elements in $\Lambda$. If $\Lambda=\{k, \ldots, d\}$, then there is nothing to prove. Assume that $\Lambda \neq\{k, \ldots, d\}$ and let $l$ be the largest element of $\{k, \ldots, d\} \backslash \Lambda$. Let $a$ be an element of the left hand side of (3.3.3). Then

$$
\begin{aligned}
a & \in\left(y_{1}, \ldots, y_{u-1}, x_{l}, x_{\lambda} \mid \lambda \in \Lambda\right) M: y_{u} \\
& =\left(y_{1}, \ldots, y_{u-1}, x_{l}, x_{\lambda} \mid \lambda \in \Lambda\right) M
\end{aligned}
$$

by the induction hypothesis. We put $a=x_{l} b+c$ with

$$
c \in\left(y_{1}, \ldots, y_{u-1}, x_{\lambda} \mid \lambda \in \Lambda\right) M .
$$

Since $x_{l} \in \mathfrak{a}\left(M / \mathfrak{q}_{l+1} M\right)$ or $x_{l} \in \mathfrak{a}(M)$,

$$
\begin{aligned}
b & \in\left(y_{1}, \ldots, y_{u-1}, x_{\lambda} \mid \lambda \in \Lambda\right) M: y_{u} x_{l} \\
& =\left(y_{1}, \ldots, y_{u-1}, x_{\lambda} \mid \lambda \in \Lambda\right) M: x_{l} .
\end{aligned}
$$

Therefore we have $a \in\left(y_{1}, \ldots, y_{u-1}, x_{\lambda} \mid \lambda \in \Lambda\right) M$. By letting $\Lambda=\emptyset$, we obtain (3.3.1).

Next we prove (3.3.2) by descending induction on $i$. We may assume that $n_{i}=$ $\cdots=n_{j}=1$ by using $\left(B_{i j}\right)$ of Theorem 3.1 and (3.3.1). In the case of $i=j,(3.3 .2)$ is included in (3.3.3).

Assume that $i<j$ and let $a$ be an element of the left hand side of (3.3.2). By the induction hypothesis, we have

$$
\begin{aligned}
a & \in\left[\left(y_{1}, \ldots, y_{u-1}, x_{i}, x_{\lambda} \mid \lambda \in \Lambda\right) M+\mathfrak{q}_{i+1}^{2} \cdots \mathfrak{q}_{j} M\right]: y_{u} \\
& =\left(y_{1}, \ldots, y_{u-1}, x_{i}, x_{\lambda} \mid \lambda \in \Lambda\right) M+\mathfrak{q}_{i+1}^{2} \cdots \mathfrak{q}_{j} M .
\end{aligned}
$$

Therefore

$$
\begin{aligned}
a \in & {\left[\left(y_{1}, \ldots, y_{u-1}, x_{\lambda} \mid \lambda \in \Lambda\right) M+\mathfrak{q}_{i} \cdots \mathfrak{q}_{j} M\right]: y_{u} } \\
& \cap\left[\left(y_{1}, \ldots, y_{u-1}, x_{i}, x_{\lambda} \mid \lambda \in \Lambda\right) M+\mathfrak{q}_{i+1}^{2} \cdots \mathfrak{q}_{j} M\right] \\
= & {\left[\left(y_{1}, \ldots, y_{u-1}, x_{\lambda} \mid \lambda \in \Lambda\right) M+\mathfrak{q}_{i} \cdots \mathfrak{q}_{j} M\right]: y_{u} \cap x_{i} M } \\
& +\left(y_{1}, \ldots, y_{u-1}, x_{\lambda} \mid \lambda \in \Lambda\right) M+\mathfrak{q}_{i+1}^{2} \cdots \mathfrak{q}_{j} M \\
= & x_{i}\left\{\left[\left(y_{1}, \ldots, y_{u-1}, x_{\lambda} \mid \lambda \in \Lambda\right) M+\mathfrak{q}_{i+1} \cdots \mathfrak{q}_{j} M\right]: y_{u}\right\} \\
& +\left(y_{1}, \ldots, y_{u-1}, x_{\lambda} \mid \lambda \in \Lambda\right) M+\mathfrak{q}_{i+1}^{2} \cdots \mathfrak{q}_{j} M \\
= & \left(y_{1}, \ldots, y_{u-1}, x_{\lambda} \mid \lambda \in \Lambda\right) M+\mathfrak{q}_{i} \cdots \mathfrak{q}_{j} M .
\end{aligned}
$$

Here we used $\left(D_{i j}\right)$ of Theorem 3.1 and the induction hypothesis to show the second and the last equations, respectively. 


\section{Macaulayfication of Affine schemes}

In this section, we construct a Macaulayfication of the affine scheme $X=\operatorname{Spec} A$ if $A$ possesses a dualizing complex and is equidimensional. In this case the nonCohen-Macaulay locus $V$ of $X$ is closed and $A$ has a p-standard system of parameters of type $s$ where $s$ is an arbitrary integer such that $\operatorname{dim} V \leq s<\operatorname{dim} X$.

Let $\mathfrak{b}$ be an ideal of $A$ and $M$ an $A$-module. We agree that $R(\mathfrak{b})$ denotes the Rees algebra $\bigoplus_{n \geq 0} \mathfrak{b}^{n}$ and $R_{M}(\mathfrak{b})$ denotes the $R(\mathfrak{b})$-module $\bigoplus_{n \geq 0} \mathfrak{b}^{n} M$.

Theorem 4.1. Let $M$ be a finitely generated A-module of dimension $d>0$ and $x_{1}, \ldots, x_{d}$ a p-standard system of parameters of type $s$ for $M$. Let

$$
\begin{aligned}
\mathfrak{q}_{i} & =\left(x_{i}, \ldots, x_{d}\right) ; \\
\mathfrak{b}_{s, i} & =\mathfrak{q}_{i} \cdots \mathfrak{q}_{s+1} ; \\
Y_{s, i} & =\operatorname{Proj} R\left(\mathfrak{b}_{s, i}\right)
\end{aligned}
$$

and $\mathcal{F}_{s, i}$ be the coherent sheaf $\left[R_{M}\left(\mathfrak{b}_{s, i}\right)\right]^{\sim}$ on $Y_{s, i}$ for all $1 \leq i \leq s+1$. Then

$$
\operatorname{depth}\left(\mathcal{F}_{s, t}\right)_{p} \geq d-t+1 \text { for all closed points } p \text { on } Y_{s, t}
$$

for all $1 \leq t \leq s+1$. In particular, $\mathcal{F}_{s, 1}$ is Cohen-Macaulay.

Furthermore, if $M / \mathfrak{q}_{t} M$ is Cohen-Macaulay for some $1<t \leq s+1$, then $\mathcal{F}_{s, t}$ is already Cohen-Macaulay.

Corollary 4.2. We assume that $A$ is equidimensional and $d=\operatorname{dim} A>0$. Let $x_{1}, \ldots, x_{d}$ be a p-standard system of parameters of type $s$ for $A$ and put

$$
\begin{aligned}
\mathfrak{q}_{i} & =\left(x_{i}, \ldots, x_{d}\right) ; \\
\mathfrak{b}_{s, i} & =\mathfrak{q}_{i} \cdots \mathfrak{q}_{s+1} ; \\
Y_{s, i} & =\operatorname{Proj} R\left(\mathfrak{b}_{s, i}\right)
\end{aligned}
$$

for all $1 \leq i \leq s+1$. Then the blowing-up $f_{s, 1}: Y_{s, 1} \rightarrow X=\operatorname{Spec} A$ is a Macaulayfication of $X$.

If $A / \mathfrak{q}_{t}$ is a Cohen-Macaulay ring for some $1<t \leq s+1$, then the blowing-up $f_{s, t}: Y_{s, t} \rightarrow X$ is a Macaulayfication of $X$.

With notation above we obtain the sequence of blowing-ups, mentioned in Section 1 .

$$
Y_{s, 1} \stackrel{g_{s, 1}}{\longrightarrow} Y_{s, 2} \stackrel{g_{s, 2}}{\longrightarrow} \cdots \stackrel{g_{s, s-1}}{\longrightarrow} Y_{s, s} \stackrel{g_{s, s}}{\longrightarrow} Y_{s, s+1} \stackrel{f_{s, s+1}}{\longrightarrow} X
$$

where the center of $f_{s, s+1}$ is $\mathfrak{q}_{s+1} \mathcal{O}_{X}$ and the one of $g_{s, i}$ is $\mathfrak{q}_{i} \mathcal{O}_{Y_{s, i+1}}$ for all $i \leq s$. See [19, pp. 132-133]. Furthermore, $\mathfrak{q}_{i} \mathcal{O}_{Y_{s, i}}$ is invertible.

To prove Theorem 4.1, we show the following lemma by induction on $t$.

Lemma 4.3. Let $x_{t}, \ldots, x_{d}$ be a subsystem of parameters for $M$, where $1 \leq t<$ $d=\operatorname{dim} M$. We fix an integer $s$ such that $t-1 \leq s<d$ and put

$$
\begin{aligned}
\mathfrak{q}_{i} & =\left(x_{i}, \ldots, x_{d}\right) ; \\
\mathfrak{b}_{i} & =\mathfrak{q}_{i} \cdots \mathfrak{q}_{s+1} ; \\
Y_{i} & =\operatorname{Proj} R\left(\mathfrak{b}_{i}\right)
\end{aligned}
$$

for all $t \leq i \leq s+1$. Let $\mathcal{F}_{i}$ be the coherent sheaf $\left[R_{M}\left(\mathfrak{b}_{i}\right)\right]^{\sim}$ on $Y_{i}$ for all $t \leq i \leq$ $s+1$. If the subsystem of parameters satisfies the following three conditions:

1. the sequence $x_{i}, \ldots, x_{d}$ is a $d$-sequence on $M /\left(x_{\lambda}^{n_{\lambda}} \mid \lambda \in \Lambda\right) M$ for any positive integers $t \leq i<s+1, n_{t}, \ldots, n_{i-1}$ and $\Lambda \subseteq\{t, \ldots, i-1\}$; 
2. the sequence $x_{s+1}, \ldots, x_{d}$ is a $d^{+}$-sequence on $M /\left(x_{\lambda}^{n_{\lambda}} \mid \lambda \in \Lambda\right) M$ for any positive integers $n_{t}, \ldots, n_{s}$ and $\Lambda \subseteq\{t, \ldots, s\}$;

3. the equality

$$
\left[\left(x_{\lambda}^{n_{\lambda}} \mid \lambda \in \Lambda\right) M+\mathfrak{b}_{i}^{n_{i}} M\right]: x_{i-1}^{n_{i-1}}=\left[\left(x_{\lambda}^{n_{\lambda}} \mid \lambda \in \Lambda\right) M+\mathfrak{b}_{i}^{n_{i}} M\right]: \mathfrak{q}_{i-1}
$$

holds for any positive integers $t+1 \leq i \leq s+1, n_{t}, \ldots, n_{i}$ and $\Lambda \subseteq$ $\{t, \ldots, i-2\}$,

then

$$
\operatorname{depth}\left(\mathcal{F}_{t}\right)_{p} \geq d-t+1 \quad \text { for all closed points } p \text { on } Y_{t} .
$$

It should be mentioned that the first and the third conditions say nothing if $t=s+1$.

Proof. First we note that $\left(\mathcal{F}_{i}\right)_{p} \neq 0$ and $\operatorname{dim}\left(\mathcal{F}_{i}\right)_{p}=\operatorname{dim} M$ for all closed points $p$ on $Y_{i}$ and $t \leq i \leq s+1$. Indeed, let $\mathfrak{p}$ be an associated prime ideal of $M$ such that $\operatorname{dim} A / \mathfrak{p}=\operatorname{dim} M$. Then $\mathfrak{p}^{*}=\bigoplus_{n \geq 0} \mathfrak{p} \cap \mathfrak{b}_{i}^{n}$ is an associated prime ideal of $R_{M}\left(\mathfrak{b}_{i}\right)$ : See [31, Proposition 1.1 (iii)]. Since $x_{i}, \ldots, x_{d}$ is a subsystem of parameters for $A / \mathfrak{p}$, and hence is analytically independent on $A / \mathfrak{p}, \mathfrak{m} \mathfrak{b}_{i}^{n}$ contains $\mathfrak{p} \cap \mathfrak{b}_{i}^{n}$ for all $n \geq 0$. That is, all closed points on $Y_{i}$ contain $\mathfrak{p}^{*}$. Furthermore $\operatorname{dim} R_{M}\left(\mathfrak{b}_{i}\right)=\operatorname{dim} R\left(\mathfrak{b}_{i}\right) / \mathfrak{p}^{*}=\operatorname{dim} A / \mathfrak{p}+1$ [31, Corollary 1.6].

Assume that $t=s+1$ and let $R=R\left(\mathfrak{b}_{s+1}\right)$. Then $\mathfrak{b}_{s+1}=\mathfrak{q}_{s+1}$ is generated by a $d^{+}$-sequence on $M$ of length $d-s$. We find that, for all $0 \leq i \leq d-s$, the $i^{\text {th }}$ local cohomology module of $R_{M}\left(\mathfrak{b}_{s+1}\right)$ with respect to $\mathfrak{q}_{s+1} R+R_{+}$is annihilated by some power of $R_{+}$by [15, Proposition 4.9], whose brief proof is included in Appendix [A] By passing through the completion of $A$ and using [ㅁ, Satz 1], we obtain (4.3.1).

We note that we can obtain (4.3.1) without the theory of $d^{+}$-sequences if $s=$ $d-1$. Indeed, it is easy to see $Y_{d} \cong \operatorname{Spec} A / H_{x_{d}}^{0}(A)$ and $\mathcal{F}_{d}=\left(M / H_{x_{d}}^{0}(M)\right)^{\sim}$.

Next we assume that $t \leq s$. Then $Y_{t}$ is the blowing-up of $Y_{t+1}$ with respect to $\mathfrak{q}_{t} \mathcal{O}_{Y_{t+1}}$. Let $q$ be a closed point on $Y_{t}$ and $p$ its image on $Y_{t+1}$ under the blowing-up $Y_{t} \rightarrow Y_{t+1}$. Then $p$ is also a closed point. Let $B=\mathcal{O}_{Y_{t+1}, p}, N=\left(\mathcal{F}_{t+1}\right)_{p}$ and $\mathfrak{n}$ be the maximal ideal of $B$. Since $\mathfrak{q}_{s+1} \mathcal{O}_{Y_{t+1}}$ is invertible, $\mathfrak{q}_{s+1} B$ is generated by $x_{i}$ for some $t+1 \leq i \leq d$. In this case $x_{i}$ is a regular element on $B$ and on $N$. Thus we have the expression of $\mathcal{O}_{Y_{t}, q}$ and $\left(\mathcal{F}_{t}\right)_{q}$ :

$$
\mathcal{O}_{Y_{t}, q}=B\left[x_{i} / x_{t}\right]_{\left(\mathfrak{n}, f\left(x_{i} / x_{t}\right)\right)}, \quad\left(\mathcal{F}_{t}\right)_{q}=\left[\bigcup_{n>0} \frac{\left(x_{t}, x_{i}\right)^{n} N}{x_{t}^{n}}\right]_{\left(\mathfrak{n}, f\left(x_{i} / x_{t}\right)\right)}
$$

or

$$
\mathcal{O}_{Y_{t}, q}=B\left[x_{t} / x_{i}\right]_{\left(\mathfrak{n}, f\left(x_{t} / x_{i}\right)\right)}, \quad\left(\mathcal{F}_{t}\right)_{q}=\left[\bigcup_{n>0} \frac{\left(x_{t}, x_{i}\right)^{n} N}{x_{i}^{n}}\right]_{\left(\mathfrak{n}, f\left(x_{t} / x_{i}\right)\right)}
$$

where $f$ is a monic polynomial with coefficient in $B$.

We compute the local cohomology $H_{\mathfrak{q}_{t}}^{p}(N)$ of $N$ with respect to $\mathfrak{q}_{t}$. Since $\mathfrak{q}_{t} B=$ $\left(x_{i}, x_{t}\right) B, H_{\mathfrak{q}_{t}}^{q}(N)=0$ if $q>2$. Furthermore $H_{\mathfrak{q}_{t}}^{0}(N)=0$ because $x_{i}$ is regular on $N$. Let $\mathcal{F}_{t+1}^{(l)}$ be the coherent sheaf $\left[R_{M / x_{t}^{l} M}\left(\mathfrak{b}_{t+1}\right)\right]^{\sim}$ on $Y_{t+1}$ and $N^{(l)}=\left(\mathcal{F}_{t+1}^{(l)}\right)_{p}$. Then the induction hypothesis says that

$$
\operatorname{depth} N, \operatorname{depth} N^{(l)} \geq d-t .
$$


There exists a short exact sequence

$$
0 \rightarrow \bigoplus_{n>0} \frac{\mathfrak{b}_{t+1}^{n} M: x_{t}^{l}}{\mathfrak{b}_{t+1}^{n} M+0:{ }_{M} x_{t}^{l}} \stackrel{x_{t}^{l}}{\longrightarrow} \bigoplus_{n>0} \frac{\mathfrak{b}_{t+1}^{n} M}{x_{t}^{l} \mathfrak{b}_{t+1}^{n} M} \rightarrow \bigoplus_{n>0} \frac{\mathfrak{b}_{t+1}^{n} M+x_{t}^{l} M}{x_{t}^{l} M} \rightarrow 0
$$

The left hand side of (4.3.4) is annihilated by $\mathfrak{q}_{t}$ because of (3). On the other hand, $x_{i}$ is regular on the right hand side of (4.3.4). Indeed, if $i \leq s$, then $x_{i}, \ldots, x_{d}$ is a $d$-sequence on $M / x_{t}^{l} M$ and hence

$$
x_{t}^{l} M: x_{i} \cap\left[x_{t}^{l} M+\mathfrak{b}_{t+1}^{n} M\right] \subseteq x_{t}^{l} M: x_{i} \cap\left(x_{t}^{l}, x_{i}, \ldots, x_{d}\right) M=x_{t}^{l} M .
$$

If $i \geq s+1$, then $x_{s+1}, \ldots, x_{d}$ is a $d^{+}$-sequence on $M / x_{t}^{l} M$. Therefore

$$
x_{t}^{l} M: x_{i} \cap\left[x_{t}^{l} M+\mathfrak{b}_{t+1}^{n} M\right] \subseteq x_{t}^{l} M: x_{i} \cap\left(x_{t}^{l}, x_{s+1}, \ldots, x_{d}\right) M=x_{t}^{l} M .
$$

By taking local cohomology with respect to $x_{i}$ of (4.3.4), we obtain

$$
H_{x_{i}}^{1}\left(\bigoplus_{n>0} \frac{\mathfrak{b}_{t+1}^{n} M}{x_{t}^{l} \mathfrak{b}_{t+1}^{n} M}\right)=H_{x_{i}}^{1}\left(\bigoplus_{n>0} \frac{\mathfrak{b}_{t+1}^{n} M+x_{t}^{l} M}{x_{t}^{l} M}\right)
$$

and

$$
H_{x_{i}}^{0}\left(\bigoplus_{n>0} \frac{\mathfrak{b}_{t+1}^{n} M}{x_{t}^{l} \mathfrak{b}_{t+1}^{n} M}\right)=\bigoplus_{n>0} \frac{\mathfrak{b}_{t+1}^{n} M: x_{t}^{l}}{\mathfrak{b}_{t+1}^{n} M+0: M x_{t}^{l}} .
$$

Taking localization of (4.3.5) at $p$, we have

$$
H_{x_{i}}^{1}\left(N / x_{t}^{l} N\right)=H_{x_{i}}^{1}\left(N^{(l)}\right)=\operatorname{inj} \lim _{m} N^{(l)} / x_{i}^{m} N^{(l)} .
$$

The spectral sequence $E_{2}^{p q}=H_{x_{i}}^{p} H_{x_{t}}^{q}(-) \Rightarrow H_{\left(x_{t}, x_{i}\right)}^{n}(-)$ induces a short exact sequence

$$
0 \rightarrow H_{x_{i}}^{1} H_{x_{t}}^{p-1}(-) \rightarrow H_{\left(x_{t}, x_{i}\right)}^{p}(-) \rightarrow H_{x_{i}}^{0} H_{x_{t}}^{p}(-) \rightarrow 0
$$

Hence

$$
\begin{aligned}
H_{\mathfrak{q}_{t}}^{2}(N) & =H_{x_{i}}^{1} H_{x_{t}}^{1}(N) \\
& =H_{x_{i}}^{1}\left(\operatorname{inj}{ }_{l} \lim N / x_{t}^{l} N\right) \\
& =\operatorname{inj} \lim _{l} H_{x_{i}}^{1}\left(N / x_{t}^{l} N\right) \\
& =\operatorname{inj} \lim _{l} H_{x_{i}}^{1}\left(N^{(l)}\right) \\
& =i \operatorname{inj}_{m, l} N^{(l)} / x_{i}^{m} N^{(l)} .
\end{aligned}
$$

Since $x_{i}$ is regular on $N^{(l)}$ and $\operatorname{depth} N^{(l)} \geq d-t$,

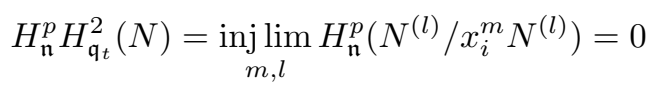

for $p<d-t-1$.

Next we compute $H_{\mathfrak{q}_{t}}^{1}(N)$. Since $x_{t}, \ldots, x_{d}$ is a $d$-sequence,

$$
0:{ }_{M} x_{t} \cap \mathfrak{b}_{t+1}^{n} M \subseteq 0:{ }_{M} x_{t} \cap \mathfrak{q}_{t} M=0
$$

if $n>0$. Therefore $x_{t}$ is regular on $\bigoplus_{n>0} \mathfrak{b}_{t+1}^{n} M$ and hence on $N$, that is $H_{x_{t}}^{0}(N)=$ 0. From (4.3.7), we have

$$
H_{\mathfrak{q}_{t}}^{1}(N)=H_{x_{i}}^{0} H_{x_{t}}^{1}(N)=\operatorname{inj} \lim _{l} H_{x_{i}}^{0}\left(N / x_{t}^{l} N\right) .
$$


Taking localization and direct limit of [4.3.6), we find that $\mathfrak{q}_{t} H_{\mathfrak{q}_{t}}^{1}(N)=0$.

We consider the spectral sequence $E_{2}^{p q}=H_{\mathfrak{n}}^{p} H_{\mathfrak{q} t}^{q}(N) \Rightarrow H_{\mathfrak{n}}^{n}(N)$. We already know that $E_{2}^{p q}=0$ if $q>2$ or $q=0$ and that $E_{2}^{p 2}=0$ if $p<d-t-1$. Since $\operatorname{depth} N \geq d-t, E_{2}^{p 1}=H_{\mathfrak{n}}^{p+1}(N)=0$ for $p<d-t-1$. Thus we obtain that

$$
H_{\mathfrak{n}}^{p} H_{\mathfrak{q}_{t}}^{q}(N)=0 \quad \text { if } q \neq 1,2 \text { or } p<d-t-1
$$

and

$$
\mathfrak{q}_{t} H_{\mathfrak{q}_{t}}^{1}(N)=0 .
$$

By using this, we compute the depth of $\left(\mathcal{F}_{t}\right)_{q}$. We assume that it has the expression (4.3.2). Let $L=N[T] /\left(x_{t} T-x_{i}\right) N[T]$, where $T$ is an indeterminate and $N[T]=B[T] \otimes N$. Then

$$
\bigcup_{n>0} \frac{\left(x_{t}, x_{i}\right)^{n} N}{x_{t}^{n}} \cong L / H_{x_{t}}^{0}(L)
$$

Taking local cohomology with respect to $\mathfrak{q}_{t}$ of the short exact sequence

$$
0 \rightarrow N[T] \stackrel{x_{t} T-x_{i}}{\longrightarrow} N[T] \rightarrow L \rightarrow 0
$$

we obtain an exact sequence

$$
0 \rightarrow H_{\mathfrak{q}_{t}}^{1}(N[T]) \rightarrow H_{\mathfrak{q}_{t}}^{1}(L) \rightarrow H_{\mathfrak{q}_{t}}^{2}(N[T]) \rightarrow H_{\mathfrak{q}_{t}}^{2}(N[T]) \rightarrow 0 .
$$

Recall that $H_{\mathfrak{q}_{t}}^{1}(N[T])=H_{\mathfrak{q}_{t}}^{1}(N) \otimes B[T]$ is annihilated by $\mathfrak{q}_{t}$ and hence by $x_{t} T-x_{i}$. By using an exact sequence

$$
0 \rightarrow H_{f(T)}^{1} H_{\mathfrak{n}}^{p-1}(-) \rightarrow H_{(\mathfrak{n}, f(T))}^{p}(-) \rightarrow H_{f(T)}^{0} H_{\mathfrak{n}}^{p}(-) \rightarrow 0,
$$

we get $H_{(\mathfrak{n}, f(T))}^{p} H_{\mathfrak{q}_{t}}^{q}(N[T])=0$ if $q \neq 1,2$ or $p<d-t$. Indeed, the monic polynomial $f(T)$ is regular on $H_{\mathfrak{n}}^{p} H_{\mathfrak{q}_{t}}^{q}(N[T])=\left(H_{\mathfrak{n}}^{p} H_{\mathfrak{q}_{t}}^{q}(N)\right) \otimes B[T]$. Hence we obtain that

$$
H_{(\mathfrak{n}, f(T))}^{p} H_{\mathfrak{q}_{t}}^{1}(L)=0 \quad \text { for } p<d-t
$$

Taking local cohomology with respect to $\mathfrak{q}_{t} B[T]=\left(x_{t}, x_{t} T-x_{i}\right) B[T]$ of a short exact sequence,

$$
0 \rightarrow H_{x_{t}}^{0}(L) \rightarrow L \rightarrow L / H_{x_{t}}^{0}(L) \rightarrow 0
$$

we have

$$
H_{\mathfrak{q}_{t}}^{1}\left(L / H_{x_{t}}^{0}(L)\right) \cong H_{\mathfrak{q}_{t}}^{1}(L)
$$

that is,

$$
H_{(\mathfrak{n}, f(T))}^{p} H_{\mathfrak{q}_{t}}^{1}\left(L / H_{x_{t}}^{0}(L)\right)=0 \quad \text { for } p<d-t .
$$

Of course, $H_{\mathfrak{q}_{t}}^{q}\left(L / H_{x_{t}}^{0}(L)\right)=0$ if $q \neq 1$. The spectral sequence

$$
E_{2}^{p q}=H_{(\mathfrak{n}, f(T))}^{p} H_{\mathfrak{q}_{t}}^{q}\left(L / H_{x_{t}}^{0}(L)\right) \Rightarrow H_{(\mathfrak{n}, f(T))}^{n}\left(L / H_{x_{t}}^{0}(L)\right)
$$

says that $\operatorname{depth}\left(\mathcal{F}_{t}\right)_{q} \geq d-t+1$. In the case of (4.3.3), we can also show that $\operatorname{depth}\left(\mathcal{F}_{t}\right)_{q} \geq d-t+1$. The proof is completed.

Now we return to Theorem 4.1 . 
Proof of Theorem 4.1. Because of Proposition 2.8. Corollaries 2.10 and 3.2] the subsystem of parameters $x_{t}, \ldots, x_{d}$ satisfies the assumption of Lemma 4.3 for any $1 \leq t \leq s+1$. Hence we obtain 4.1.1.

Next we assume that $t \geq 2$ and that $M / \mathfrak{q}_{t} M$ is Cohen-Macaulay, that is, $x_{1}, \ldots$, $x_{t-1}$ is a regular sequence on $M / \mathfrak{q}_{t} M$. Corollary 3.3 says that $x_{1}, \ldots, x_{t-1}$ is a regular sequence on $M / \mathfrak{b}_{s, t}^{n} M$ for any $n>0$ and $M$ itself. Taking Koszul cohomology of the short exact sequence

$$
0 \rightarrow R_{M}\left(\mathfrak{b}_{s, t}\right) \rightarrow \bigoplus_{n \geq 0} M_{n} \rightarrow \bigoplus_{n>0} M / \mathfrak{b}_{s, t}^{n} M \rightarrow 0
$$

where $M_{n}$ denotes a copy of $M$, with respect to $x_{1}, \ldots, x_{t-1}$, we obtain that

$$
H^{t-1}\left(x_{1}, \ldots, x_{t-1} ; R_{M}\left(\mathfrak{b}_{s, t}\right)\right)=R_{M /\left(x_{1}, \ldots, x_{t-1}\right) M}\left(\mathfrak{b}_{s, t}\right)
$$

and

$$
H^{i}\left(x_{1}, \ldots, x_{t-1} ; R_{M}\left(\mathfrak{b}_{s, t}\right)\right)=0 \text { for all } i<t-1 .
$$

Let $\mathcal{F}_{s, t}^{\prime}=\left[R_{M /\left(x_{1}, \ldots, x_{t-1}\right) M}\left(\mathfrak{b}_{s, t}\right)\right]^{\sim}$ and $p$ be a closed point on $Y_{s, t}$. Two equations above mean that $x_{1}, \ldots, x_{t-1}$ is a regular sequence on $\left(\mathcal{F}_{s, t}\right)_{p}$ and

$$
\left(\mathcal{F}_{s, t}^{\prime}\right)_{p}=\left(\mathcal{F}_{s, t}\right)_{p} /\left(x_{1}, \ldots, x_{t-1}\right)\left(\mathcal{F}_{s, t}\right)_{p}
$$

Lemma 4.3 also assures us that $\operatorname{depth}\left(\mathcal{F}_{s, t}^{\prime}\right)_{p} \geq d-t+1$. Therefore $\mathcal{F}_{s, t}$ is CohenMacaulay. The proof of Theorem 4.1 is completed.

Proof of Corollary 4.2. We already know the depth of $Y_{s, t}$ at each closed point. Since $\operatorname{dim} A / \mathfrak{b}_{s, t}=s<\operatorname{dim} A$, there exists no minimal prime ideal of $A$ containing $\mathfrak{b}_{s, t}$. Therefore $f_{s, t}$ is birational. Of course, the projective morphism $f_{s, t}$ is proper. Thus the proof is completed.

Next we consider an $R\left(\mathfrak{b}_{s, i+1}^{2}\right)$-module $R_{\mathfrak{b}_{s, i+1}\left[\mathfrak{b}_{s, i+1} M: x_{i}\right]}\left(\mathfrak{b}_{s, i+1}^{2}\right)$ when $s>0$. It is well-known that $Y_{s, i+1} \cong \operatorname{Proj} R\left(\mathfrak{b}_{s, i+1}^{2}\right)$. We identify them.

Lemma 4.4. With the same notation as Theorem 4.1, let $\mathcal{G}_{s, i}$ be the coherent sheaf $\left[R_{\mathfrak{b}_{s, i+1}\left[\mathfrak{b}_{s, i+1} M: x_{i}\right]}\left(\mathfrak{b}_{s, i+1}^{2}\right)\right]^{\sim}$ on $Y_{s, i+1}$ for all $1 \leq i \leq s$. Then

$$
\operatorname{depth}\left(\mathcal{G}_{s, t}\right)_{p} \geq d-t+1 \quad \text { for all closed points } p \text { on } Y_{s, t+1}
$$

for all $1 \leq t \leq s$. In particular $\mathcal{G}_{s, 1}$ is Cohen-Macaulay.

Proof. We first show that $x_{t}, x_{t+1}$ is a regular sequence on $\mathfrak{b}_{s, t+1}^{2 n-1}\left[\mathfrak{b}_{s, t+1} M: x_{t}\right]$ for all $n>0$. Indeed,

$$
0:{ }_{M} x_{t} \cap \mathfrak{b}_{s, t+1}^{2 n-1}\left[\mathfrak{b}_{s, t+1} M: x_{t}\right] \subseteq 0:{ }_{M} x_{t} \cap \mathfrak{q}_{t} M=0 .
$$

Let $a$ be an element of $\mathfrak{b}_{s, t+1}^{2 n-1}\left[\mathfrak{b}_{s, t+1} M: x_{t}\right]$ such that

$$
x_{t+1} a \in x_{t} \mathfrak{b}_{s, t+1}^{2 n-1}\left[\mathfrak{b}_{s, t+1} M: x_{t}\right] .
$$

Then $x_{t} a \in x_{t}^{2} M: x_{t+1} \cap \mathfrak{b}_{s, t+1}^{2 n} M \subset x_{t}^{2} M$ because $x_{t+1}, \ldots, x_{d}$ is a $d$-sequence on $M / x_{t}^{2} M$. Let $x_{t} a=x_{t}^{2} b$. Then

$$
b \in \mathfrak{b}_{s, t+1}^{2 n} M: x_{t}^{2}=\mathfrak{b}_{s, t+1}^{2 n-1}\left[\mathfrak{b}_{s, t+1} M: x_{t}\right]+0:{ }_{M} x_{t}
$$


because of Theorem 3.1 Hence

$$
\begin{aligned}
a & \in\left\{x_{t} \mathfrak{b}_{s, t+1}^{2 n-1}\left[\mathfrak{b}_{s, t+1} M: x_{t}\right]+0:{ }_{M} x_{t}\right\} \cap \mathfrak{b}_{s, t+1}^{2 n-1} M \\
& =x_{t} \mathfrak{b}_{s, t+1}^{2 n-1}\left[\mathfrak{b}_{s, t+1} M: x_{t}\right]+0:{ }_{M} x_{t} \cap \mathfrak{b}_{s, t+1}^{2 n-1} M \\
& =x_{t} \mathfrak{b}_{s, t+1}^{2 n-1}\left[\mathfrak{b}_{s, t+1} M: x_{t}\right] .
\end{aligned}
$$

Next we consider the short exact sequence of $R\left(\mathfrak{b}_{s, t+1}^{2}\right)$-modules

$$
0 \rightarrow \bigoplus_{n>0} \mathfrak{b}_{s, t+1}^{2 n} M \rightarrow \bigoplus_{n>0} \mathfrak{b}_{s, t+1}^{2 n-1}\left[\mathfrak{b}_{s, t+1} M: x_{t}\right] \rightarrow \bigoplus_{n>0} \frac{\mathfrak{b}_{s, t+1}^{2 n-1}\left[\mathfrak{b}_{s, t+1} M: x_{t}\right]}{\mathfrak{b}_{s, t+1}^{2 n} M} \rightarrow 0 .
$$

Since the right hand side of 4.4.2) is annihilated by $\mathfrak{q}_{t}$ and $x_{t}, x_{t+1}$ is a regular sequence on the middle term of (4.4.2), we obtain that

$$
H_{\mathfrak{q}_{t}}^{q}\left(\bigoplus_{n>0} \mathfrak{b}_{s, t+1}^{2 n-1}\left[\mathfrak{b}_{s, t+1} M: x_{t}\right]\right)= \begin{cases}H_{\mathfrak{q}_{t}}^{q}\left(\bigoplus_{n>0} \mathfrak{b}_{s, t+1}^{2 n} M\right) & \text { if } q \geq 2 \\ 0 & \text { if } q=0 \text { or } 1 .\end{cases}
$$

Let $p$ be a closed point on $Y_{s, t+1}$. Then by taking localization of the equation above at $p$, we have

$$
H_{\mathfrak{q}_{t}}^{q}\left(\left(\mathcal{G}_{s, t}\right)_{p}\right)= \begin{cases}H_{\mathfrak{q}_{t}}^{q}\left(\left(\mathcal{F}_{s, t+1}\right)_{p}\right) & \text { if } q \geq 2 \\ 0 & \text { if } q=0 \text { or } 1 .\end{cases}
$$

We know that $H_{\mathfrak{q}_{t}}^{q}\left(\left(\mathcal{F}_{s, t+1}\right)_{p}\right)=0$ for $q>2$ and $H_{\mathfrak{n}}^{p} H_{\mathfrak{q}_{t}}^{2}\left(\left(\mathcal{F}_{s, t+1}\right)_{p}\right)=0$ for all $p<d-t-1$. Here $\mathfrak{n}$ denotes the maximal ideal of $\mathcal{O}_{Y_{s, t+1}, p}$. The spectral sequence $E_{2}^{p q}=H_{\mathfrak{n}}^{p} H_{\mathfrak{q}_{t}}^{q}(-) \Rightarrow H_{\mathfrak{n}}^{n}(-)$ says (4.4.1).

Corollary 4.5. With the same notation as Corollary 4.2 we put

$$
Z_{s, i}=\operatorname{Proj} R\left(\mathfrak{b}_{s, i+1}\left[\mathfrak{b}_{s, i+1}: x_{i}\right]\right)
$$

for all $1 \leq i \leq s$. Then

$$
\operatorname{depth} \mathcal{O}_{Z_{s, t}, p} \geq d-t+1 \quad \text { for all closed points } p \text { on } Z_{s, t}
$$

for all $1 \leq t \leq s$. In particular the blowing-up $Z_{s, 1} \rightarrow X$ is a Macaulayfication of $X$.

The schemes $Z_{s, s}$ and $Z_{s, s-1}$ were essentially given by Brodmann [4, Satz 9.2] and the author [21, Theorem 5.6], respectively.

Proof. Since

$$
\mathfrak{b}_{s, t+1}^{n}\left[\mathfrak{b}_{s, t+1}: x_{t}\right]^{n} \subseteq \mathfrak{b}_{s, t+1}^{n}\left[\mathfrak{b}_{s, t+1}^{n}: x_{t}^{n}\right] \subseteq \mathfrak{b}_{s, t+1}^{2 n-1}\left[\mathfrak{b}_{s, t+1}: x_{t}\right]
$$

for all $n>0, \mathfrak{b}_{s, t+1}^{2}$ is a reduction of $\mathfrak{b}_{s, t+1}\left[\mathfrak{b}_{s, t+1}: x_{t}\right]$. Hence we obtain a finite morphism $h_{s, t}: Z_{s, t} \rightarrow Y_{s, t+1}$. Let $p$ be a closed point on $Y_{s, t+1}$. Then Lemma 4.4 assures us that $\operatorname{depth}\left(\left(h_{s, t}\right)_{*} \mathcal{O}_{Z_{s, t}}\right)_{p} \geq d-t+1$. Hence we obtain 4.5.1). We can show that the blowing-up $Z_{s, t} \rightarrow X$ is birational in the same way as Corollary 4.2

These Macaulayfications are different from each other. We find it by comparing the height and the analytic spread [23] of centers.

Lemma 4.6. With the same notation as Corollary 4.2, the analytic spread of $\mathfrak{b}_{s, i}$ is equal to $d-i+1$ for all $1 \leq i \leq s+1$. Furthermore, the one of $\mathfrak{b}_{s, i+1}\left[\mathfrak{b}_{s, i+1}: x_{i}\right]$ is equal to $d-i$ for all $1 \leq i \leq s$. 
Proof. The ideal $\mathfrak{b}_{s, i}$ is generated by monomials of $x_{i}, \ldots, x_{d}$ of degree $s-i+2$. Since $x_{i}, \ldots, x_{d}$ is analytically independent,

$$
\operatorname{length}\left(\mathfrak{q}_{i}^{n} / \mathfrak{m} \mathfrak{q}_{i}^{n}\right) \leq \operatorname{length}\left(\mathfrak{b}_{s, i}^{n} / \mathfrak{m} \mathfrak{b}_{s, i}^{n}\right) \leq \operatorname{length}\left(\mathfrak{q}_{i}^{n(s-i+2)} / \mathfrak{m} \mathfrak{q}_{i}^{n(s-i+2)}\right)
$$

for all $n>0$. Thus the first assertion is proved.

Since $\mathfrak{b}_{s, i+1}^{2}$ is a reduction of $\mathfrak{b}_{s, i+1}\left[\mathfrak{b}_{s, i+1}: x_{i}\right]$, they have the same analytic spread.

\section{Macaulayfication of Noetherian schemes}

We prove Theorem 1.1 in this section. First we consider a Macaulayfication of quasi-projective schemes.

Theorem 5.1. Let $A$ be a Noetherian ring possessing a dualizing complex and $X$ a quasi-projective scheme over $A$. Then there exists a proper morphism $f: Y \rightarrow X$ with an open dense subset $U$ of $X$ such that

1. $Y$ is Cohen-Macaulay;

2. $f^{-1}(U)$ is dense in $Y$;

3. $\left.f\right|_{f^{-1}(U)}: f^{-1}(U) \rightarrow U$ is an isomorphism.

That is, $f$ is a Macaulayfication of $X$.

If finitely many Cohen-Macaulay points $p_{1}, \ldots, p_{n}$ on $X$ are given, then we may choose $f$ and $Y$ such that

4. $U$ contains $p_{1}, \ldots, p_{n}$.

Since a quasi-projective scheme is an open dense subscheme of a projective scheme, we may consider only projective schemes without loss of generality. Let $R=\bigoplus_{n>0} R_{n}$ be a Noetherian graded ring such that $R_{0}$ has a dualizing complex and $R$ is generated by $R_{1}$ as an $R_{0}$-algebra. We give a Macaulayfication of $X=\operatorname{Proj} R$. Of course, we assume that $X$ is not Cohen-Macaulay and let $V$ be the non-Cohen-Macaulay locus of $X$.

In this case $R$ possesses a dualizing complex $D^{\bullet}$ with codimension function $t$ as a graded ring.

The following lemma is an analogue of Lemma 2.4 and can be proved by the local duality theorem.

Lemma 5.2. Let $M$ be a finitely generated graded $R$-module and $\mathfrak{p}$ a homogeneous prime ideal of $R$.

1. If $\mathfrak{p} \supseteq \prod_{j>i}$ ann $H^{j}\left(\operatorname{Hom}\left(M, D^{\bullet}\right)\right)$, then $t(\mathfrak{p})>i$;

2. Assume that $t(\mathfrak{q})=0$ for all minimal prime ideals $\mathfrak{q}$ of $R$. Then $M_{\mathfrak{p}}$ is Cohen-Macaulay if and only if $\mathfrak{p} \nsupseteq \prod_{j>0}$ ann $H^{j}\left(\operatorname{Hom}\left(M, D^{\bullet}\right)\right)$.

Since $\operatorname{Proj} R / H_{R_{+}}^{0}(R) \cong X$, we may assume that $H_{R_{+}}^{0}(R)=0$. Furthermore, we may assume that $t$ is constant on the associated prime ideals of $R$. Indeed, let $(0)=\mathfrak{q}_{1} \cap \cdots \cap \mathfrak{q}_{n}$ be the primary decomposition of the zero ideal of $R$. Of course, $\mathfrak{q}_{i}$ is homogeneous. For any integer $i$, let $\mathfrak{r}_{i}$ be the intersection of $\mathfrak{q}_{j}$ such that $\mathfrak{q}_{j}$ is an isolated component and $t\left(\sqrt{\mathfrak{q}_{j}}\right)=i$. Then

$$
g: \coprod_{i} \operatorname{Proj} R / \mathfrak{r}_{i} \rightarrow X
$$

is birational and proper. Indeed, $\mathfrak{r}_{i}=R$ for all but finitely many $i$, hence $g$ is a finite morphism. On the other hand, $g$ is an isomorphism on the Cohen-Macaulay 
locus $X \backslash V$ of $X$ because a Cohen-Macaulay local ring is equidimensional and has no embedded prime ideal. If each $\operatorname{Proj} R / \mathfrak{r}_{i}$ has a Macaulayfication, then $X$ also has a Macaulayfication. Therefore we assume that $t(\mathfrak{p})=0$ for all associated prime ideals $\mathfrak{p}$. In this case $t(\mathfrak{p})=h t \mathfrak{p}$ for any prime ideal.

Since $X$ has a dualizing complex, $\operatorname{dim} X<\infty$. Let $d=\operatorname{dim} X$ and $s$ be an integer such that $d-\operatorname{codim}(V, X) \leq s<d$. It should be mentioned that $d-\operatorname{codim}(V, X) \neq$ $\operatorname{dim} V$ in general.

The following lemma is an easy consequence of prime avoidance. See the proof of Theorem 2.7 .

Lemma 5.3. With notation above, there exists a sequence $z_{1}, \ldots, z_{d}$ of homogeneous elements in $R$ satisfying the following statements:

1. if $\mathfrak{p}$ is a minimal prime ideal of $R /\left(z_{i}, \ldots, z_{d}\right) R$ but not containing $R_{+}$, then ht $\mathfrak{p}=d-i+1$

2. $z_{s+1}, \ldots, z_{d} \in \prod_{j>0}$ ann $H^{j}\left(D^{\bullet}\right)$;

3. $z_{i} \in \prod_{j>d-i}$ ann $H^{j}\left(\operatorname{Hom}\left(R /\left(z_{i+1}, \ldots, z_{d}\right) R, D^{\bullet}\right)\right.$ for all $i \leq s$.

Let $\mathfrak{b}=\prod_{i=1}^{s+1}\left(z_{i}, \ldots, z_{d}\right)$ where $z_{1}, \ldots, z_{d}$ is a sequence obtained above and $f: Y \rightarrow X$ be the blowing-up of $X$ with respect to $\mathfrak{b}^{\sim}$. We show that $f$ is a Macaulayfication of $X$. Since no minimal prime ideal of $R$ contains $z_{d}$ and hence $\mathfrak{b}, f$ is birational and proper.

Let $q$ be a closed point on $Y$ and $\mathfrak{p} \subset R$ its image under $f$. If $\mathfrak{b} \nsubseteq \mathfrak{p}$, then $\prod_{j>0}$ ann $H^{j}\left(D^{\bullet}\right) \not \mathfrak{p}$. Hence $\mathcal{O}_{Y, q} \cong R_{(\mathfrak{p})}$ is Cohen-Macaulay.

Assume that $\mathfrak{b} \subseteq \mathfrak{p}$. Then $z_{t}, \ldots, z_{d} \in \mathfrak{p}$ and $z_{t-1} \notin \mathfrak{p}$ for some $1 \leq t \leq s+1$, where we set $z_{0}=1$. Choose an element $y \in R_{1} \backslash \mathfrak{p}$ and let $x_{i}=z_{i} / y^{\operatorname{deg} z_{i}}$. Then

1. $\operatorname{dim} R_{(\mathfrak{p})} /\left(x_{t}, \ldots, x_{d}\right) R_{(\mathfrak{p})}=\operatorname{dim} R_{(\mathfrak{p})}-(d-t+1)$;

2. $x_{s+1}, \ldots, x_{d} \in \mathfrak{a}\left(R_{(\mathfrak{p})}\right)$;

3. $x_{i} \in \mathfrak{a}\left(R_{(\mathfrak{p})} /\left(x_{i+1}, \ldots, x_{d}\right) R_{(\mathfrak{p})}\right)$ for all $t \leq i \leq s$.

4. $\mathfrak{a}\left(R_{(\mathfrak{p})} /\left(x_{t}, \ldots, x_{d}\right) R_{(\mathfrak{p})}\right)=R_{(\mathfrak{p})}$ if $t>1$.

Hence $x_{t}, \ldots, x_{d}$ is a subsystem of p-standard system of parameters for $R_{(\mathfrak{p})}$ and $R_{(\mathfrak{p})} /\left(x_{t}, \ldots, x_{d}\right) R_{(\mathfrak{p})}$ is a Cohen-Macaulay ring if $t>1$. Since

$$
\mathfrak{b} R_{(\mathfrak{p})}=\prod_{i=t}^{s+1}\left(x_{i}, \ldots, x_{d}\right) R_{(\mathfrak{p})},
$$

Corollary 4.2 says that $\mathcal{O}_{Y, q}$ is Cohen-Macaulay.

Let $\mathfrak{p}_{1}, \ldots, \mathfrak{p}_{n}$ be finitely many Cohen-Macaulay points on $X$. Then we may choose $z_{d}$ such that $z_{d} \notin \mathfrak{p}_{1} \cup \cdots \cup \mathfrak{p}_{n}$. Thus the proof of Theorem 5 .1] is completed.

Proof of Theorem 1.1] Let $A$ be a Noetherian ring possessing a dualizing complex and $X$ a separated, of finite type scheme over $\operatorname{Spec} A$. By using Chow's Lemma [16] Théorème 5.6.1], we obtain a birational proper morphism $X^{\prime} \rightarrow X$ such that $X^{\prime}$ is a quasi-projective scheme over $\operatorname{Spec} A$. Theorem 5.1 assures us that there exists a Macaulayfication $Y \rightarrow X^{\prime}$ of $X^{\prime}$. The composition $Y \rightarrow X^{\prime} \rightarrow X$ is a Macaulayfication of $X$. 


\section{SHARP'S CONJECTURE}

We divide Theorem 1.2 into two statements.

Theorem 6.1. Let $A$ be a Noetherian ring possessing a dualizing complex with codimension function $t$. If

1. $t(\mathfrak{p})-\mathrm{ht} \mathfrak{p}$ is locally constant on $\operatorname{Spec} A$;

2. A has no embedding prime ideal,

then $A$ is a homomorphic image of finite-dimensional Gorenstein ring.

Of course, an integral domain satisfies conditions (1) and (2).

Proof. Let $D^{\bullet}$ be a dualizing complex of $A$. If $\operatorname{Spec} A$ is disconnected, then $A$ is a direct product of Noetherian rings which also satisfy (11) and (21). Therefore we may assume that $\operatorname{Spec} A$ is connected. In this case, condition (1) is equivalent to

$1^{\prime} A_{\mathfrak{m}}$ is equidimensional for all maximal ideals $\mathfrak{m}$ of $A$.

We assume that $t(\mathfrak{p})=\mathrm{ht} \mathfrak{p}$ for all prime ideals $\mathfrak{p}$ of $A$ instead of (1).

Let $V$ be the non-Cohen-Macaulay locus of $X=\operatorname{Spec} A$. We work by induction on $s(A)=\operatorname{dim} A-\operatorname{codim}(V, X)$. Let $d=\operatorname{dim} A$ and $s=s(A)$. It should be mentioned that a Noetherian ring possessing a dualizing complex is of finite dimension.

Assume that $s>d$, that is, $A$ is Cohen-Macaulay and let $K=H^{0}\left(D^{\bullet}\right)$. Then the idealization $A \ltimes K$ is a Gorenstein ring and $A$ is its homomorphic image. Indeed, a prime ideal of $A \ltimes K$ has an expression $\mathfrak{p} \oplus K$ where $\mathfrak{p}$ is a prime ideal of $A$. Since $K_{\mathfrak{p}}=H^{0}\left(D_{\mathfrak{p}}^{\bullet}\right)$ is the canonical module of $A_{\mathfrak{p}},[A \ltimes K]_{\mathfrak{p} \oplus K}=A_{\mathfrak{p}} \ltimes K_{\mathfrak{p}}$ is a Gorenstein local ring [25].

Next we assume that $s \leq d$. In the same way as the preceding section, there exists a sequence $x_{1}, \ldots, x_{d}$ of elements in $A$ such that

1. ht $\left(x_{i}, \ldots, x_{d}\right)=d-i+1$ for all $i$;

2. $x_{s+1}, \ldots, x_{d} \in \prod_{j>0}$ ann $H^{j}\left(D^{\bullet}\right)$;

3. $x_{i} \in \prod_{j>d-i}$ ann $H^{j}\left(\operatorname{Hom}\left(A /\left(x_{i+1}, \ldots, x_{d}\right), D^{\bullet}\right)\right)$ for $1 \leq i \leq s$.

Let $\mathfrak{b}=\prod_{i=1}^{s+1}\left(x_{i}, \ldots, x_{d}\right)$ and $R=R\left(\mathfrak{b}^{d-s-1}\right)$. Then a finitely generated $A$ algebra $R$ has a dualizing complex.

We show that $R$ satisfies $\left(1^{\prime}\right)$. Let $\mathfrak{M}$ be a maximal ideal of $R$. Since all minimal prime ideals are homogeneous, we may assume that $\mathfrak{M}$ is homogeneous. Let $\mathfrak{m}=$ $\mathfrak{M} \cap A$. Then $\mathfrak{M}=\mathfrak{m} R+R_{+}$. We may assume that $A$ is a local ring with maximal ideal $\mathfrak{m}$ by passing though the localization. If $\mathfrak{b} \nsubseteq \mathfrak{m}$, then $R_{\mathfrak{M}} \cong A[T]_{(\mathfrak{m}, T)}$, where $T$ is an indeterminate, and it is equidimensional. Assume that $\mathfrak{b} \subseteq \mathfrak{m}$. Let $\mathfrak{p}_{1}, \ldots, \mathfrak{p}_{m}$ be the associated prime ideals of $A$ and $\mathfrak{p}_{i}^{*}=\bigoplus_{n>0} \mathfrak{b}^{n(d-s-1)} \cap \mathfrak{p}_{i}$. Then $\mathfrak{p}_{1}^{*}, \ldots, \mathfrak{p}_{m}^{*}$ are the associated prime ideals of $R$ and $\operatorname{dim} R / \mathfrak{p}_{i}^{*}=\operatorname{dim} A / \mathfrak{p}_{i}+1=d+1$ for all $i$. See Proposition 1.1 and Corollary 1.6 of [31]. In particular, we find $\operatorname{dim} R=d+1$. Proposition 1.1 of [31] also assures us that $R$ satisfies (2).

Next we show that $R$ is Cohen-Macaulay or $s(R)<s$. Assume that $R$ is not Cohen-Macaulay and let $\mathfrak{P}$ be a prime ideal of $R$ such that $R_{\mathfrak{P}}$ is not CohenMacaulay and ht $\mathfrak{P}=(d+1)-s(R)$. Then $\mathfrak{P}$ must be homogeneous. By the same argument as the preceding section, we have $\mathfrak{P} \supseteq \mathfrak{b}^{d-s-1} R+R_{+}$. Let $\mathfrak{p}=\mathfrak{P} \cap A$. Then $\mathfrak{P}=\mathfrak{p} R+R_{+}$and ht $\mathfrak{P}=$ ht $\mathfrak{p}+1$. Since $\mathfrak{p} \supseteq\left(x_{s+1}, \ldots, x_{d}\right)$, the height of $\mathfrak{p}$ must be at least $d-s$. If ht $\mathfrak{p}=d-s$, then $x_{s} \notin \mathfrak{p}$. Since $x_{s+1}, \ldots, x_{d}$ is a $d^{+}$-sequence on $A_{\mathfrak{p}}, R_{\mathfrak{p}}=R\left(\left(x_{s+1}, \ldots, x_{d}\right)^{d-s-1} A_{\mathfrak{p}}\right)$ is Cohen-Macaulay. See 
15, Theorem 7.11] or Theorem A.5 in appendix A. It is a contradiction. Hence $s(R)=(d+1)-$ ht $\mathfrak{P}=d-$ ht $\mathfrak{p}<s$. In particular, if $s=0$, then $R$ must be Cohen-Macaulay.

The induction hypothesis says that $R$ is a homomorphic image of a finitedimensional Gorenstein ring and hence $A \cong R / R_{+}$is also. Thus the proof is completed.

Corollary 6.2. A Noetherian local ring possessing a dualizing complex is a homomorphic image of a Gorenstein local ring.

Proof. Let $A$ be a Noetherian local ring possessing a dualizing complex. By [1. Lemma 3.1], we may assume that $A$ satisfies Serre's $\left(S_{2}\right)$-condition. On the other hand, Ogoma [24, Lemma 4.1] showed that such a ring is equidimensional and has no embedded prime ideal. Therefore $A$ satisfies the assumption of Theorem 6.1

\section{Acknowledgment}

The author thanks Professor Shiro Goto and all members of the seminar on Commutative Algebra at Meiji University for many helpful discussion during this research. This article was written when the author visited Germany. He is grateful to Professor Manfred Hermann and all members of his working group on Commutative Algebra at the University of Cologne.

\section{Appendix A. $d^{+}$-SEQUences}

In this appendix, we select a few results from [15]. The author would like to thank Professor Shiro Goto and Professor Kikumichi Yamagishi for permission to refer to their results.

Let $A$ be a commutative ring, $M$ an $A$-module and $x_{1}, \ldots, x_{s}$ a $d^{+}$-sequence on $M$. We put $\mathfrak{q}=\left(x_{1}, \ldots, x_{s}\right)$.

Theorem A.1 ([15, Lemma 2.2]). Assume that $s \geq 2$. For any integers $n_{1}, \ldots$, $n_{s-1} \geq 2$, we have

$$
\left(x_{1}^{n_{1}}, \ldots, x_{s-1}^{n_{s-1}}\right) M: x_{s}=\sum_{\Lambda \subseteq\{1, \ldots, s-1\}}\left(\prod_{\lambda \in \Lambda} x_{\lambda}^{n_{\lambda}-1}\right)\left[\left(x_{\lambda} \mid \lambda \in \Lambda\right) M: x_{s}\right] .
$$

Here we set $\left(x_{\lambda} \mid \lambda \in \Lambda\right)=(0)$ and $\prod_{\lambda \in \Lambda} x_{\lambda}^{n_{\lambda}-1}=1$ if $\Lambda=\emptyset$.

Proof. We work by induction on $s$. Let $a \in x_{1}^{n_{1}} M: x_{2}$ and put $x_{2} a=x_{1}^{n_{1}} b$. Then $b \in x_{2} M: x_{1}^{n_{1}}=x_{2} M: x_{1}$. If we put $x_{1} b=x_{2} a^{\prime}$, then $a^{\prime} \in x_{1} M: x_{2}$ and $a-$ $x_{1}^{n_{1}-1} a^{\prime} \in 0:{ }_{M} x_{2}$. Hence $a \in x_{1}^{n_{1}-1}\left[x_{1} M: x_{2}\right]+0:{ }_{M} x_{2}$.

Assume that $s>2$. Since $x_{s-1}, x_{s}$ is a $d^{+}$-sequence on $M /\left(x_{1}^{n_{1}}, \ldots, x_{s-2}^{n_{s-2}}\right) M$,

$$
\begin{aligned}
\left(x_{1}^{n_{1}}, \ldots, x_{s-1}^{n_{s-1}}\right) M: x_{s}= & x_{s-1}^{n_{s-1}-1}\left[\left(x_{1}^{n_{1}}, \ldots, x_{s-2}^{n_{s-2}}, x_{s-1}\right) M: x_{s}\right] \\
& +\left(x_{1}^{n_{1}}, \ldots, x_{s-2}^{n_{s-2}}\right) M: x_{s} .
\end{aligned}
$$


Since $x_{1}, \ldots, x_{s-2}, x_{s}$ is a $d^{+}$-sequence on $M / x_{s-1} M$ and on $M$ itself, we obtain

$$
\begin{aligned}
& \left(x_{1}^{n_{1}}, \ldots, x_{s-1}^{n_{s-1}}\right) M: x_{s} \\
= & x_{s-1}^{n_{s-1}-1}\left\{\sum_{\Lambda \subseteq\{1, \ldots, s-2\}}\left(\prod_{\lambda \in \Lambda} x_{\lambda}^{n_{\lambda}-1}\right)\left[\left(x_{s-1}, x_{\lambda} \mid \lambda \in \Lambda\right) M: x_{s}\right]\right\} \\
& +\sum_{\Lambda \subseteq\{1, \ldots, s-2\}}\left(\prod_{\lambda \in \Lambda} x_{\lambda}^{n_{\lambda}-1}\right)\left[\left(x_{\lambda} \mid \lambda \in \Lambda\right) M: x_{s}\right] \\
= & \sum_{\Lambda \subseteq\{1, \ldots, s-1\}}\left(\prod_{\lambda \in \Lambda} x_{\lambda}^{n_{\lambda}-1}\right)\left[\left(x_{\lambda} \mid \lambda \in \Lambda\right) M: x_{s}\right]
\end{aligned}
$$

by the induction hypothesis.

Theorem A.2 ([15, Theorem 2.6]). For any integers $1 \leq k \leq s, n \geq 0$ and $n_{1}, \ldots, n_{k} \geq 1$, we have

$$
\left(x_{1}^{n_{1}}, \ldots, x_{k}^{n_{k}}\right) M \cap \mathfrak{q}^{n} M=\sum_{i=1}^{k} x_{i}^{n_{i}} \mathfrak{q}^{n-n_{i}} M
$$

Proof. We prove $\left(A_{k}\right)$ and

$$
\begin{aligned}
& {\left[\left(x_{1}^{n_{1}}, \ldots, x_{k-1}^{n_{k-1}}\right) M: x_{k}+x_{k} M\right] \cap \mathfrak{q}^{n} M} \\
& =\sum_{i=1}^{k-1} x_{i}^{n_{i}} \mathfrak{q}^{n-n_{i}} M+x_{k} \mathfrak{q}^{n-1} M \\
& \quad+\sum_{\substack{\Lambda \subseteq\{1, \ldots, k-1\} \\
\sum_{\lambda \in \Lambda}\left(n_{\lambda}-1\right) \geq n}}\left(\prod_{\lambda \in \Lambda} x_{\lambda}^{n_{\lambda}-1}\right)\left[\left(x_{\lambda} \mid \lambda \in \Lambda\right) M: x_{k}\right]
\end{aligned}
$$

for $1 \leq k \leq s$ by induction on $k$. Here we set $\sum_{\lambda \in \Lambda}\left(n_{\lambda}-1\right)=0$ if $\Lambda=\emptyset$.

First we show $\left(A_{1}\right)$ by induction on $n_{1}$. Lemma 2.2 says that $x_{1} M \cap \mathfrak{q}^{n} M=$ $x_{1} \mathfrak{q}^{n-1} M$ for all $n \geq 0$. Assume that $n>n_{1}>1$ and let $x_{1}^{n_{1}} a \in \mathfrak{q}^{n} M$. Then $x_{1}^{n_{1}} a \in x_{1}^{n_{1}-1} M \cap \mathfrak{q}^{n} M=x_{1}^{n_{1}-1} \mathfrak{q}^{n-n_{1}+1} M$ by the induction hypothesis. Let $x_{1}^{n_{1}} a=$ $x_{1}^{n_{1}-1} a^{\prime}$ with $a^{\prime} \in \mathfrak{q}^{n-n_{1}+1} M$. Then $x_{1} a-a^{\prime} \in 0:{ }_{M} x_{1} \cap \mathfrak{q} M=0$, that is, $a^{\prime}=x_{1} a$. Therefore $a^{\prime} \in x_{1} M \cap \mathfrak{q}^{n-n_{1}+1} M=x_{1} \mathfrak{q}^{n-n_{1}} M$ and hence $x_{1}^{n_{1}} a \in x_{1}^{n_{1}} \mathfrak{q}^{n-n_{1}} M$. If $n_{1} \geq n$, then $\left(A_{1}\right)$ is trivial.

Next we show $\left(B_{1}\right)$. If $n=0$, then it is trivial. Assume that $n>0$. Let $a$ be an element of the left hand side. Then $x_{1} a \in x_{1}^{2} M \cap \mathfrak{q}^{n+1} M=x_{1}^{2} \mathfrak{q}^{n-1} M$. If we put $x_{1} a=x_{1}^{2} b$ with $b \in \mathfrak{q}^{n-1} M$, then $a-x_{1} b \in 0:{ }_{M} x_{1} \cap \mathfrak{q}^{n} M=0$. Therefore $a \in x_{1} \mathfrak{q}^{n-1} M$.

Assume that $k \geq 2$. We show $\left(B_{k}\right)$ by induction on $n$. If $n=0$, then $\left(B_{k}\right)$ coincides with Theorem A.1 Let $n>0$. If $n_{i}=1$, then $\left(B_{k}\right)$ comes from $\left(B_{k-1}\right)$ applied to a $d^{+}$-sequence $x_{1}, \ldots, x_{i-1}, x_{i+1}, \ldots, x_{s}$ on $M / x_{i} M$. For example, if 
$n_{1}=1$, then

$$
\begin{aligned}
{\left[\left(x_{1}, x_{2}^{n_{2}}, \ldots, x_{k-1}^{n_{k-1}}\right) M: x_{k}+x_{k} M\right] \cap\left[x_{1} M+\mathfrak{q}^{n} M\right] } \\
=x_{1} M+\sum_{i=2}^{k-1} x_{i}^{n_{i}} \mathfrak{q}^{n^{-n_{i}}} M+x_{k} \mathfrak{q}_{k}^{n_{k}-1} M \\
\quad+\sum_{\substack{\Lambda \subseteq\{2, \ldots, k-1\} \\
\sum_{\lambda \in \Lambda}\left(n_{\lambda}-1\right) \geq n}}\left(\prod_{\lambda \in \Lambda} x_{\lambda}^{n_{\lambda}-1}\right)\left[\left(x_{1}, x_{\lambda} \mid \lambda \in \Lambda\right) M: x_{k}\right] .
\end{aligned}
$$

Taking intersection with $\mathfrak{q}^{n} M$, we obtain $\left(B_{1}\right)$. Thus we may assume that $n_{1}, \ldots$, $n_{k-1} \geq 2$. Let $a$ be an element of the left hand side of $\left(B_{k}\right)$. Since $a \in \mathfrak{q}^{n-1} M$,

$$
\begin{aligned}
& a \in\left[\left(x_{1}^{n_{1}}, \ldots, x_{k-1}^{n_{k-1}}\right) M: x_{k}+x_{k} M\right] \cap \mathfrak{q}^{n-1} M \\
& =\sum_{i=1}^{k-1} x_{i}^{n_{i}} \mathfrak{q}^{n-n_{i}-1} M+x_{k} \mathfrak{q}^{n-2} M \\
& +\sum_{\substack{\Lambda \subseteq\{1, \ldots, k-1\} \\
\sum_{\lambda \in \Lambda}\left(n_{\lambda}-1\right) \geq n-1}}\left(\prod_{\lambda \in \Lambda} x_{\lambda}^{n_{\lambda}-1}\right)\left[\left(x_{\lambda} \mid \lambda \in \Lambda\right) M: x_{k}\right] .
\end{aligned}
$$

We put

$$
a=b+\sum_{\substack{\Lambda \subseteq\{1, \ldots, k-1\} \\ \sum_{\lambda \in \Lambda}\left(n_{\lambda}-1\right) \geq n-1}}\left(\prod_{\lambda \in \Lambda} x_{\lambda}^{n_{\lambda}-1}\right) c_{\Lambda}
$$

with $b \in \sum_{i=1}^{k-1} x_{i}^{n_{i}} \mathfrak{q}^{n-n_{i}-1} M+x_{k} \mathfrak{q}^{n-2} M$ and $c_{\Lambda} \in\left(x_{\lambda} \mid \lambda \in \Lambda\right) M: x_{k}$. If $\sum_{\lambda \in \Lambda}\left(n_{\lambda}-1\right)=n-1$, then $\left(\prod_{\lambda \in \Lambda} x_{\lambda}^{n_{\lambda}-1}\right) c_{\Lambda} \in\left(x_{\lambda}^{n_{\lambda}} \mid \lambda \in \Lambda\right) M$. Indeed, by renumbering $x_{1}, \ldots, x_{k-1}$, we may assume that $\Lambda=\{1, \ldots, p\}$. Then

$$
a \in \mathfrak{q}^{n} M \subset\left(x_{1}^{n_{1}}, \ldots, x_{p}^{n_{p}}, x_{p+1}, \ldots, x_{s}\right) M .
$$

Let $\Lambda^{\prime} \subset\{1, \ldots, k-1\}$ such that $\sum_{\lambda \in \Lambda^{\prime}}\left(n_{\lambda}-1\right) \geq n-1$. If $\Lambda^{\prime} \subsetneq \Lambda$, then

$$
\sum_{\lambda \in \Lambda^{\prime}}\left(n_{\lambda}-1\right)<\sum_{\lambda \in \Lambda}\left(n_{\lambda}-1\right)=n-1,
$$

which is a contradiction. Therefore if $\Lambda^{\prime} \neq \Lambda$, then $\Lambda^{\prime} \cap\{p+1, \ldots, k-1\} \neq \emptyset$ and hence $\left(\prod_{\lambda \in \Lambda^{\prime}} x_{\lambda}^{n_{\lambda}-1}\right) c_{\Lambda^{\prime}} \in\left(x_{p+1}, \ldots, x_{k-1}\right) M$. On the other hand, it is easy to show that $\left(x_{1}^{n_{1}-1} \cdots x_{p}^{n_{p}-1}\right) c_{\Lambda} \in\left(x_{1}^{n_{1}}, \ldots, x_{p}^{n_{p}}\right) M: x_{k}$. Therefore.

$$
\begin{aligned}
\left(x_{1}^{n_{1}-1} \cdots x_{p}^{n_{p}-1}\right) c_{\Lambda} & =a-b-\sum_{\Lambda^{\prime} \neq \Lambda}\left(\prod_{\lambda \in \Lambda} x_{\lambda}^{n_{\lambda}-1}\right) c_{\Lambda^{\prime}} \\
& \in\left(x_{1}^{n_{1}}, \ldots, x_{p}^{n_{p}}\right) M: x_{k} \cap\left(x_{1}^{n_{1}}, \ldots, x_{p}^{n_{p}}, x_{p+1}, \ldots, x_{s}\right) M \\
& =\left(x_{1}^{n_{1}}, \ldots, x_{p}^{n_{p}}\right) M .
\end{aligned}
$$


Here we applied Lemma 2.2 to a $d$-sequence $x_{1}^{n_{1}}, \ldots, x_{p}^{n_{p}}, x_{k}, x_{p+1}, \ldots, x_{k-1}$, $x_{k+1}, \ldots, x_{s}$ on $M$. Thus

$$
\begin{aligned}
a-\sum_{\sum_{\lambda \in \Lambda}\left(n_{\lambda}-1\right) \geq n}\left(\prod_{\lambda \in \Lambda} x_{\lambda}^{n_{\lambda}-1}\right) c_{\Lambda} & =b+\sum_{\sum_{\lambda \in \Lambda}\left(n_{\lambda}-1\right)=n-1}\left(\prod_{\lambda \in \Lambda} x_{\lambda}^{n_{\lambda}-1}\right) c_{\Lambda} \\
& \in\left(x_{1}^{n_{1}}, \ldots, x_{k-1}^{n_{k-1}}, x_{k}\right) M \cap \mathfrak{q}^{n} M \\
& =\left(x_{1}^{n_{1}}, \ldots, x_{k-1}^{n_{k}-1}, x_{k}\right) M \\
& \cap\left[\mathfrak{q}^{n} M+x_{k} M\right] \cap \mathfrak{q}^{n} M \\
= & {\left[\sum_{i=1}^{k-1} x_{i}^{n_{i}} \mathfrak{q}^{n-n_{i}} M+x_{k} M\right] \cap \mathfrak{q}^{n} M } \\
& =\sum_{i=1}^{k-1} x_{i}^{n_{i}} \mathfrak{q}^{n-n_{i}} M+x_{k} \mathfrak{q}^{n-1} M .
\end{aligned}
$$

Here we applied $\left(A_{k-1}\right)$ to a $d^{+}$-sequence $x_{1}, \ldots, x_{k-1}, x_{k+1}, \ldots, x_{s}$ on $M / x_{k} M$. Thus $\left(B_{k}\right)$ is proved.

Next we show $\left(A_{k}\right)$ by induction on $n_{k}$. If $n_{k}=1$, then we obtain $\left(A_{k}\right)$ by applying $\left(A_{k-1}\right)$ to a $d^{+}$-sequence $x_{1}, \ldots, x_{k-1}, x_{k+1}, \ldots, x_{s}$ on $M / x_{k} M$. Assume that $n_{k}>1$ and let $a \in\left(x_{1}^{n_{1}}, \ldots, x_{k}^{n_{k}}\right) M \cap \mathfrak{q}^{n} M$. Then

$$
\begin{aligned}
a & \in\left(x_{1}^{n_{1}}, \ldots, x_{k-1}^{n_{k-1}}, x_{k}^{n_{k}-1}\right) M \cap \mathfrak{q}^{n} M \\
& =\sum_{i=1}^{k-1} x_{i}^{n_{i}} \mathfrak{q}^{n-n_{i}} M+x_{k}^{n_{k}-1} \mathfrak{q}^{n-n_{k}+1} M .
\end{aligned}
$$

Let $a=b+x_{k}^{n_{k}-1} c$ with $b \in \sum_{i=1}^{k-1} x_{i}^{n_{i}} \mathfrak{q}^{n-n_{i}} M$ and $c \in \mathfrak{q}^{n-n_{k}+1} M$. Then

$$
c \in\left(x_{1}^{n_{1}}, \ldots, x_{k}^{n_{k}}\right) M: x_{k}^{n_{k}-1} .
$$

If we put $x_{k}^{n_{k}-1} c=x_{k}^{n_{k}} c^{\prime}+d$ with $d \in\left(x_{1}^{n_{1}}, \ldots, x_{k-1}^{n_{k-1}}\right) M$, then

$$
\begin{aligned}
c-x_{k} c^{\prime} & \in\left(x_{1}^{n_{1}}, \ldots, x_{k-1}^{n_{k-1}}\right) M: x_{k}^{n_{k}-1} \\
& =\left(x_{1}^{n_{1}}, \ldots, x_{k-1}^{n_{k-1}}\right) M: x_{k} .
\end{aligned}
$$

Therefore

$$
c \in\left(x_{1}^{n_{1}}, \ldots, x_{k-1}^{n_{k-1}}\right) M: x_{k}+x_{k} M .
$$

By using $\left(B_{k}\right)$, we obtain

$$
\begin{aligned}
c \in & {\left[\left(x_{1}^{n_{1}}, \ldots, x_{k-1}^{n_{k-1}}\right) M: x_{k}+x_{k} M\right] \cap \mathfrak{q}^{n-n_{k}+1} M } \\
= & \sum_{i=1}^{k-1} x_{i}^{n_{i}} \mathfrak{q}^{n-n_{k}-n_{i}+1} M+x_{k} \mathfrak{q}^{n-n_{k}} M \\
& +\sum_{\substack{\Lambda \subseteq\{1, \ldots, k-1\} \\
\sum_{\lambda \in \Lambda}\left(n_{\lambda}-1\right) \geq n-n_{k}+1}}\left(\prod_{\lambda \in \Lambda} x_{\lambda}^{n_{\lambda}-1}\right)\left[\left(x_{\lambda} \mid \lambda \in \Lambda\right) M: x_{k}\right]
\end{aligned}
$$

and hence $a=b+x_{k}^{n_{k}-1} c \in \sum_{i=1}^{k} x_{i}^{n_{i}} \mathfrak{q}^{n-n_{i}} M$. 
From now on, we assume that $x_{1}, \ldots, x_{s}$ is a $d^{+}$-sequence on $A$ itself. Theorems A.3 A.5 still hold if $A$ is not Noetherian. We, however, assume that $A$ is Noetherian for the sake of simplicity. We compute local cohomology modules of the Rees algebra $R(\mathfrak{q})=A[\mathfrak{q} t]$, where $t$ is an indeterminate. Let $G(\mathfrak{q})=R(\mathfrak{q}) / \mathfrak{q} R(\mathfrak{q})$.

Theorem A.3 ([15, Proposition 4.3]). If $p<s$, then

$$
\left[H_{\mathfrak{q} t}^{p}(G(\mathfrak{q}))\right]_{n}=0 \quad \text { for } n \neq-p .
$$

In particular $H_{\mathfrak{q} t}^{0}(G(\mathfrak{q}))=\left[H_{\mathfrak{q} t}^{0}(G(\mathfrak{q}))\right]_{0} \cong 0:{ }_{A} x_{1}$.

Proof. We show that

$$
\left[H_{\left(x_{1} t, \ldots, x_{q} t\right)}^{p}(G(\mathfrak{q}))\right]_{n}=0 \text { if } p<q \text { and } n \neq-p
$$

for all $1 \leq q \leq s$ by induction on $q$. Let $\overline{a t^{n}} \in H_{x_{1} t}^{0}(G(\mathfrak{q}))$, that is, $a \in \mathfrak{q}^{n}$ and $x_{1}^{l} a \in \mathfrak{q}^{n+l+1}$ for some $l>0$. Then $x_{1}^{l} a \in\left(x_{1}^{l}\right) \cap \mathfrak{q}^{n+l+1}=x_{1}^{l} \mathfrak{q}^{n+1}$. Let $x_{1}^{l} a=x_{1}^{l} a^{\prime}$ with $a^{\prime} \in \mathfrak{q}^{n+1}$. If $n>0$, then $a-a^{\prime} \in 0:{ }_{A} x_{1} \cap \mathfrak{q}=0$, that is, $\overline{a t^{n}}=0$ in $G(\mathfrak{q})$. If $n=0$, then $a \in 0:{ }_{A} x_{1}+\mathfrak{q}$. On the other hand, if $a \in 0:{ }_{A} x_{1}+\mathfrak{q}$, then $\bar{a} \in H_{x_{1} t}^{0}(G(\mathfrak{q}))$. Hence

$$
H_{x_{1} t}^{0}(G(\mathfrak{q}))=\left(0: x_{1}+\mathfrak{q}\right) / \mathfrak{q} \cong 0: x_{1} .
$$

Of course, $\left[H_{x_{1} t}^{0}(G(\mathfrak{q}))\right]_{n}=0$ if $n<0$.

Assume that $q>1$. From the spectral sequence $E_{2}^{r s}=H_{x_{q} t}^{r} H_{\left(x_{1} t, \ldots, x_{q-1} t\right)}^{s}(-) \Rightarrow$ $H_{\left(x_{1} t, \ldots, x_{q} t\right)}^{n}(-)$, we obtain an exact sequence

$$
0 \rightarrow H_{x_{q} t}^{1} H_{\left(x_{1} t, \ldots, x_{q-1} t\right)}^{p-1}(-) \rightarrow H_{\left(x_{1} t, \ldots, x_{q} t\right)}^{p}(-) \rightarrow H_{x_{q} t}^{0} H_{\left(x_{1} t, \ldots, x_{q-1} t\right)}^{p}(-) \rightarrow 0 .
$$

The induction hypothesis says that $x_{q} t$ annihilates $H_{\left(x_{1} t, \ldots, x_{q-1} t\right)}^{p}(G(\mathfrak{q}))$ for all $p<$ $q-1$. Therefore, if $p<q-1$, then

$$
H_{\left(x_{1} t, \ldots, x_{q} t\right)}^{p}(G(\mathfrak{q}))=H_{x_{q} t}^{0} H_{\left(x_{1} t, \ldots, x_{q-1} t\right)}^{p}(G(\mathfrak{q}))=H_{\left(x_{1} t, \ldots, x_{q-1} t\right)}^{p}(G(\mathfrak{q}))
$$

is concentrated in degree $-p$. Moreover

$$
\begin{aligned}
H_{\left(x_{1} t, \ldots, x_{q} t\right)}^{q-1}(G(\mathfrak{q})) & =H_{x_{q} t}^{0} H_{\left(x_{1} t, \ldots, x_{q-1} t\right)}^{q-1}(G(\mathfrak{q})) \\
& =\underset{m}{\operatorname{inj} \lim } H_{x_{q} t}^{0}\left(\frac{G(\mathfrak{q})}{\left(\left(x_{1} t\right)^{m}, \ldots,\left(x_{q-1} t\right)^{m}\right) G(\mathfrak{q})}(m(q-1))\right) \\
& =\underset{m}{\operatorname{inj} \lim } \bigcup_{l} \frac{\left(\left(x_{1} t\right)^{m}, \ldots,\left(x_{q-1} t\right)^{m}\right) G(\mathfrak{q}):\left(x_{q} t\right)^{l}}{\left(\left(x_{1} t\right)^{m}, \ldots,\left(x_{q-1} t\right)^{m}\right) G(\mathfrak{q})}(m(q-1)) .
\end{aligned}
$$

Let $\overline{a t^{n+m(q-1)}} \in\left(\left(x_{1} t\right)^{m}, \ldots,\left(x_{q-1} t\right)^{m}\right) G(\mathfrak{q}):\left(x_{q} t\right)^{l}$, that is, $a \in \mathfrak{q}^{n+m(q-1)}$ and

$$
x_{q}^{l} a \in\left(x_{1}^{m}, \ldots, x_{q-1}^{m}\right) \mathfrak{q}^{n+m(q-2)+l}+\mathfrak{q}^{n+m(q-1)+l+1} .
$$

If we put $a=b+c$ with $b \in\left(x_{1}^{m}, \ldots, x_{q-1}^{m}\right) \mathfrak{q}^{n+m(q-2)+l}$ and $c \in \mathfrak{q}^{n+m(q-1)+l+1}$, then

$$
\begin{aligned}
c & \in\left(x_{1}^{m}, \ldots, x_{q-1}^{m}, x_{q}^{l}\right) \cap \mathfrak{q}^{n+m(q-1)+l+1} \\
& =\left(x_{1}^{m}, \ldots, x_{q-1}^{m}\right) \mathfrak{q}^{n+m(q-2)+l+1}+x_{q}^{l} \mathfrak{q}^{n+m(q-1)+1} .
\end{aligned}
$$

If we put $c=b^{\prime}+x_{q}^{l} a^{\prime}$ with $b^{\prime} \in\left(x_{1}^{m}, \ldots, x_{q-1}^{m}\right) \mathfrak{q}^{n+m(q-2)+l+1}$ and $a^{\prime} \in \mathfrak{q}^{n+m(q-1)+1}$, then $\overline{a t^{n+m(q-1)}}=\overline{\left(a-a^{\prime}\right) t^{n+m(q-1)}}$. Therefore, by replacing $a$ by $a-a^{\prime}$, we may assume that $x_{q}^{l} a \in\left(x_{1}^{m}, \ldots, x_{q-1}^{m}\right) \mathfrak{q}^{n+m(q-2)+l}$. Then

$$
a \in\left(x_{1}^{m}, \ldots, x_{q-1}^{m}\right): x_{q} \cap \mathfrak{q}^{n+m(q-1)} .
$$




$$
\begin{aligned}
& \text { If } n>-q+1, \text { then } \mathfrak{q}^{n+m(q-1)} \subset\left(x_{1}^{m}, \ldots, x_{q-1}^{m}, x_{q}, \ldots, x_{s}\right) \text { and hence } \\
& \qquad \begin{aligned}
a & \in\left(x_{1}^{m}, \ldots, x_{q-1}^{m}\right): x_{q} \cap\left(x_{1}^{m}, \ldots, x_{q-1}^{m}, x_{q}, \ldots, x_{s}\right) \cap \mathfrak{q}^{n+m(q-1)} \\
& =\left(x_{1}^{m}, \ldots, x_{q-1}^{m}\right) \cap \mathfrak{q}^{n+m(q-1)} \\
& =\left(x_{1}^{m}, \ldots, x_{q-1}^{m}\right) \mathfrak{q}^{n+m(q-2)} .
\end{aligned}
\end{aligned}
$$

That is, $\overline{a t^{n+m(q-1)}} \in\left(\left(x_{1} t\right)^{m}, \ldots,\left(x_{q-1} t\right)^{m}\right) G(\mathfrak{q})$.

If $n<-q+1$, then $(m-1)(q-1) \geq n+m(q-1)+1$. Hence we have

$$
\begin{aligned}
a & \in\left(x_{1}^{m}, \ldots, x_{q-1}^{m}\right): x_{q} \\
& \subseteq \sum_{\Lambda \subsetneq\{1, \ldots, q-1\}}\left(\prod_{\lambda \in \Lambda} x_{\lambda}^{m-1}\right)\left[\left(x_{\lambda} \mid \lambda \in \Lambda\right): x_{q}\right]+\mathfrak{q}^{n+m(q-1)+1}
\end{aligned}
$$

by using Theorem A.1. Therefore

$$
\begin{aligned}
x_{1} \cdots x_{q-1} a & \in\left[\left(x_{1}^{m+1}, \ldots, x_{q-1}^{m+1}\right)+\mathfrak{q}^{n+(m+1)(q-1)+1}\right] \cap \mathfrak{q}^{n+(m+1)(q-1)} \\
& =\left(x_{1}^{m+1}, \ldots, x_{q-1}^{m+1}\right) \mathfrak{q}^{n+(m+1)(q-2)}+\mathfrak{q}^{n+(m+1)(q-1)+1} .
\end{aligned}
$$

Indeed, if $k \in\{1, \ldots, q-1\} \backslash \Lambda$, then

$$
\begin{aligned}
x_{1} \cdots x_{q-1}\left[\left(x_{\lambda} \mid \lambda \in \Lambda\right): x_{q}\right] & =x_{1} \cdots x_{q-1}\left[\left(x_{\lambda} \mid \lambda \in \Lambda\right): x_{k}\right] \\
& \subseteq\left(x_{\lambda}^{2} \mid \lambda \in \Lambda\right) .
\end{aligned}
$$

Thus we have

$$
\left(x_{1} t\right) \cdots\left(x_{q-1} t\right) \overline{a t^{n+m(q-1)}} \in\left(\left(x_{1} t\right)^{m+1}, \ldots,\left(x_{q-1} t\right)^{m+1}\right) G(\mathfrak{q}) .
$$

This implies that

$$
\begin{aligned}
\frac{G(\mathfrak{q})}{\left(\left(x_{1} t\right)^{m}, \ldots,\left(x_{q-1} t\right)^{m}\right) G(\mathfrak{q})} & (m(q-1)) \\
\stackrel{\left(x_{1} t\right) \cdots\left(x_{q} t\right)}{\longrightarrow} & \frac{G(\mathfrak{q})}{\left(\left(x_{1} t\right)^{m+1}, \ldots,\left(x_{q-1} t\right)^{m+1}\right) G(\mathfrak{q})}((m+1)(q-1))
\end{aligned}
$$

is a zero map in degree $n$. Taking direct limit, we have $\left[H_{\left(x_{1} t, \ldots, x_{q} t\right)}^{q-1}(G(\mathfrak{q}))\right]_{n}=0$ for $n<-q+1$.

Theorem A.4 ([15, Proposition 4.9]). For $1 \leq p \leq s$,

$$
\left[H_{(\mathfrak{q}, \mathfrak{q} t)}^{p}(R(\mathfrak{q}))\right]_{n}=0 \quad \text { if } n<2-p \text { or } n \geq 0 .
$$

Furthermore

$$
H_{(\mathfrak{q}, \mathfrak{q} t)}^{0}(R(\mathfrak{q}))=\left[H_{(\mathfrak{q}, \mathfrak{q} t)}^{0}(R(\mathfrak{q}))\right]_{0}=0:{ }_{A} x_{1} .
$$

Proof. We work by induction on $s$. Let $B=A / 0:{ }_{A} x_{1}$. Then $x_{1}, \ldots, x_{s}$ is also a $d^{+}$-sequence on $B$ and $x_{1}$ is $B$-regular. Furthermore,

$$
0 \rightarrow 0:{ }_{A} x_{1} \rightarrow R(\mathfrak{q}) \rightarrow R(\mathfrak{q} B) \rightarrow 0
$$

is exact because $0:{ }_{A} x_{1} \cap \mathfrak{q}^{n}=0$ if $n>0$. Therefore, $H_{(\mathfrak{q}, \mathfrak{q} t)}^{0}(R(\mathfrak{q}))$ is concentrated in degree 0 and $\left[H_{(\mathfrak{q}, \mathfrak{q} t)}^{0}(R(\mathfrak{q}))\right]_{0}=0:{ }_{A} x_{1}$. Thus we obtain (A.4.2). Furthermore, if $p>0$, then $H_{(\mathfrak{q}, \mathfrak{q} t)}^{p}(R(\mathfrak{q})) \cong H_{(\mathfrak{q}, \mathfrak{q} t)}^{p}(R(\mathfrak{q} B))$. We may assume that $0:{ }_{A} x_{1}=0$ to prove (A.4.1).

If $s=1$, then $R(\mathfrak{q}) \cong A[T]$ where $T$ is an indeterminate. Since $x_{1}, T$ is a regular sequence on $A[T], H_{(\mathfrak{q}, \mathfrak{q} t)}^{1}(R(\mathfrak{q}))=0$. 
Next we assume that $s>1$. There exist the following four short exact sequences

$$
\begin{aligned}
0 & \rightarrow R(\mathfrak{q}) \stackrel{x_{1}}{\longrightarrow} R(\mathfrak{q}) \rightarrow R(\mathfrak{q}) / x_{1} R(\mathfrak{q}) \rightarrow 0, \\
0 \rightarrow & G(\mathfrak{q})(-1) \stackrel{x_{1} t}{\longrightarrow} R(\mathfrak{q}) / x_{1} R(\mathfrak{q}) \rightarrow R\left(\mathfrak{q} / x_{1} A\right) \rightarrow 0, \\
0 & \rightarrow R(\mathfrak{q})(-1) \stackrel{x_{1} t}{\longrightarrow} R(\mathfrak{q}) \rightarrow R(\mathfrak{q}) / x_{1} t R(\mathfrak{q}) \rightarrow 0, \\
& \rightarrow A \stackrel{x_{1}}{\longrightarrow} R(\mathfrak{q}) / x_{1} t R(\mathfrak{q}) \rightarrow R\left(\mathfrak{q} / x_{1} A\right) \rightarrow 0
\end{aligned}
$$

because $\left(x_{1}\right) \cap \mathfrak{q}^{n}=x_{1} \mathfrak{q}^{n-1}$. Taking local cohomology, we obtain exact sequences

$$
\begin{gathered}
H_{(\mathfrak{q}, \mathfrak{q} t)}^{p-1}\left(R(\mathfrak{q}) / x_{1} R(\mathfrak{q})\right) \rightarrow H_{(\mathfrak{q}, \mathfrak{q} t)}^{p}(R(\mathfrak{q})) \stackrel{x_{1}}{\longrightarrow} H_{(\mathfrak{q}, \mathfrak{q} t)}^{p}(R(\mathfrak{q})), \\
H_{\mathfrak{q} t}^{p-1}(G(\mathfrak{q}))(-1) \rightarrow H_{(\mathfrak{q}, \mathfrak{q} t)}^{p-1}\left(R(\mathfrak{q}) / x_{1} R(\mathfrak{q})\right) \rightarrow H_{(\mathfrak{q}, \mathfrak{q} t)}^{p-1}\left(R\left(\mathfrak{q} / x_{1} A\right)\right), \\
H_{(\mathfrak{q}, \mathfrak{q} t)}^{p-1}\left(R(\mathfrak{q}) / x_{1} t R(\mathfrak{q})\right) \rightarrow H_{(\mathfrak{q}, \mathfrak{q} t)}^{p}(R(\mathfrak{q}))(-1) \stackrel{x_{1} t}{\longrightarrow} H_{(\mathfrak{q}, \mathfrak{q} t)}^{p}(R(\mathfrak{q})), \\
H_{\mathfrak{q}}^{p-1}(A) \rightarrow H_{(\mathfrak{q}, \mathfrak{q} t)}^{p-1}\left(R(\mathfrak{q}) / x_{1} t R(\mathfrak{q})\right) \rightarrow H_{(\mathfrak{q}, \mathfrak{q} t)}^{p-1}\left(R\left(\mathfrak{q} / x_{1} A\right)\right) .
\end{gathered}
$$

For $2 \leq p \leq s$, we have

$$
\left[H_{(\mathfrak{q}, \mathfrak{q} t)}^{p-1}\left(R\left(\mathfrak{q} / x_{1} A\right)\right)\right]_{n}=0 \quad \text { if } n<2-p+1 \text { or } n \geq 0
$$

and

$$
\left[H_{\mathfrak{q} t}^{p-1}(G(\mathfrak{q}))(-1)\right]_{n}=0 \quad \text { if } n \neq 2-p
$$

by the induction hypothesis and Theorem A.3. Therefore, if $n<2-p$ or $n \geq 0$, then we have

$$
\left[H_{(\mathfrak{q}, \mathfrak{q} t)}^{p-1}\left(R(\mathfrak{q}) / x_{1} R(\mathfrak{q})\right)\right]_{n}=0
$$

by using (A.4.4). Hence we find that

$$
\left[H_{(\mathfrak{q}, \mathfrak{q} t)}^{p}(R(\mathfrak{q}))\right]_{n} \stackrel{x_{1}}{\longrightarrow}\left[H_{(\mathfrak{q}, \mathfrak{q} t)}^{p}(R(\mathfrak{q}))\right]_{n}
$$

is a monomorphism by using A.4.3. This implies $\left[H_{(\mathfrak{q}, \mathfrak{q} t)}^{p}(R(\mathfrak{q}))\right]_{n}=0$ because $H_{(\mathfrak{q}, \mathfrak{q} t)}^{p}(R(\mathfrak{q}))$ is annihilated by some power of $x_{1}$ in elementwise.

Next we consider $H_{(\mathfrak{q}, \mathfrak{q} t)}^{1}(R(\mathfrak{q}))$. Since $H_{(\mathfrak{q} t)}^{0}(G(\mathfrak{q})) \cong H_{\mathfrak{q}}^{0}(A)=0:{ }_{A} x_{1}=0$, we have

$$
\left[H_{(\mathfrak{q}, \mathfrak{q} t)}^{0}\left(R(\mathfrak{q}) / x_{1} R(\mathfrak{q})\right)\right]_{n}=\left[H_{(\mathfrak{q}, \mathfrak{q} t)}^{0}\left(R(\mathfrak{q}) / x_{1} t R(\mathfrak{q})\right)\right]_{n}=0 \quad \text { if } n \neq 0
$$

by using (A.4.2), A.4.4) and A.4.6). Therefore we find that

$$
\left[H_{(\mathfrak{q}, \mathfrak{q} t)}^{1}(R(\mathfrak{q}))\right]_{n} \stackrel{x_{1}}{\longrightarrow}\left[H_{(\mathfrak{q}, \mathfrak{q} t)}^{1}(R(\mathfrak{q}))\right]_{n}
$$

and

$$
\left[H_{(\mathfrak{q}, \mathfrak{q} t)}^{1}(R(\mathfrak{q}))\right]_{n-1} \stackrel{x_{1} t}{\longrightarrow}\left[H_{(\mathfrak{q}, \mathfrak{q} t)}^{1}(R(\mathfrak{q}))\right]_{n}
$$

are monomorphisms for $n \neq 0$ by using (A.4.3) and (A.4.5). Hence $H_{(\mathfrak{q}, \mathfrak{q} t)}^{1}(R(\mathfrak{q}))=$ 0 because it is annihilated by some power of $x_{1}$ and $x_{1} t$ in elementwise.

The following theorem comes from Theorem A.4 and [14, Theorem 3.1.1] at once.

Theorem A.5. Let $r$ be an integer such that $r \geq s-1$. Then

$$
H_{\left(\mathfrak{q}, \mathfrak{q}^{r} t\right)}^{p}\left(R\left(\mathfrak{q}^{r}\right)\right)=0 \quad \text { for } 1 \leq p \leq s .
$$

Furthermore

$$
H_{\left(\mathfrak{q}, \mathfrak{q}^{r} t\right)}^{0}\left(R\left(\mathfrak{q}^{r}\right)\right)=\left[H_{\left(\mathfrak{q}, \mathfrak{q}^{r} t\right)}^{0}\left(R\left(\mathfrak{q}^{r}\right)\right)\right]_{0}=0:{ }_{A} x_{1}
$$




\section{Appendix B. An Example}

We give an example of a Noetherian local ring with large non-Cohen-Macaulay locus and construct its Macaulayfication according to Corollary 4.2 We used [3] to calculate our example.

Example B.1. Let $k$ be a field, $A$ the affine semigroup ring

$$
k\left[a, b, c, d, e^{2}, e^{3}, a d e, b d e, c d e, d^{2} e\right]
$$

and $\mathfrak{m}$ the homogeneous maximal ideal of $A$. Then $\operatorname{dim} A_{\mathfrak{m}}=5$ and the non-CohenMacaulay locus of $\operatorname{Spec} A_{\mathfrak{m}}$ is of dimension 3. The sequence $x_{1}=a^{4}, x_{2}=b^{4}$, $x_{3}=c^{4}, x_{4}=d^{4}, x_{5}=e^{4}$ is a p-standard system of parameters of type 3 for $A_{\mathfrak{m}}$.

Proof. Since $A$ is a finite algebra over $k\left[a, b, c, d, e^{2}\right], \operatorname{dim} A_{\mathfrak{m}}=5$ and $a, b, c, d, e^{2}$ is a system of parameters for $A_{\mathfrak{m}}$.

Next we compute $\mathfrak{a}\left(A_{\mathfrak{m}}\right)$. We regard $A$ as a graded ring in natural way. Let

$$
C=A /\left(a d, b d, c d, d^{2}, e^{2}, e^{3}, \text { ade }, b d e, c d e, d^{2} e\right) .
$$

Then there exist two short exact sequences of $A$-modules:

$$
\begin{gathered}
0 \rightarrow A \rightarrow k[a, b, c, d, e] \rightarrow C(-1) \rightarrow 0, \\
0 \rightarrow A / \mathfrak{m}(-1) \rightarrow C \rightarrow A /\left(d, e^{2}, e^{3}, \text { ade }, \text { bde }, c d e, d^{2} e\right) \rightarrow 0 .
\end{gathered}
$$

Since $A /\left(d, e^{2}, e^{3}\right.$, ade, bde, cde, $\left.d^{2} e\right) \cong k[a, b, c]$, we find that

$$
\begin{aligned}
H_{\mathfrak{m}}^{0}(C) & =A / \mathfrak{m}(-1), \\
H_{\mathfrak{m}}^{3}(C) \cong H_{(a, b, c)}^{3} & (k[a, b, c])
\end{aligned}
$$

and

$$
H_{\mathfrak{m}}^{p}(C)=0 \quad \text { if } p \neq 0,3
$$

Hence

$$
\begin{aligned}
& H_{\mathfrak{m}}^{1}(A) \cong A / \mathfrak{m}(-2), \\
& H_{\mathfrak{m}}^{4}(A) \cong H_{(a, b, c)}^{3}(k[a, b, c])(-1)
\end{aligned}
$$

and

$$
H_{\mathfrak{m}}^{p}(A)=0 \text { for } p \neq 1,4,5 \text {. }
$$

Thus we have $\mathfrak{a}\left(A_{\mathfrak{m}}\right)=\left(d, e^{2}, e^{3}\right.$, ade $\left., b d e, c d e, d^{2} e\right) \mathfrak{m} A_{\mathfrak{m}}, \operatorname{dim} A_{\mathfrak{m}} / \mathfrak{a}\left(A_{\mathfrak{m}}\right)=3$ and $d^{4}, e^{4} \in \mathfrak{a}\left(A_{\mathfrak{m}}\right)$.

To compute $\mathfrak{a}\left(A_{\mathfrak{m}} /\left(d^{4}, e^{4}\right) A_{\mathfrak{m}}\right)$, we take Koszul homologies of (B.1.1) with respect to $\left(d^{4}, e^{4}\right)$. Since $\left(d^{4}, e^{4}\right) C=0$, we obtain an exact sequence

$$
0 \rightarrow C^{2}(-5) \rightarrow A /\left(d^{4}, e^{4}\right) \rightarrow k[a, b, c, d, e] /\left(d^{4}, b^{4}\right) k[a, b, c, d, e] \rightarrow C(-1) \rightarrow 0 .
$$

Therefore

$$
\begin{aligned}
& H_{\mathfrak{m}}^{0}\left(A /\left(d^{4}, e^{4}\right)\right)=(A / \mathfrak{m})^{2}(-6), \\
& H_{\mathfrak{m}}^{1}\left(A /\left(d^{4}, e^{4}\right)\right)=A / \mathfrak{m}(-2), \\
& H_{\mathfrak{m}}^{2}\left(A /\left(d^{4}, e^{4}\right)\right)=0 .
\end{aligned}
$$

Thus $\mathfrak{a}\left(A_{\mathfrak{m}} /\left(d^{4}, e^{4}\right) A_{\mathfrak{m}}\right)=\mathfrak{m}^{2} A_{\mathfrak{m}} \ni c^{4}$. 
Lemma 2.5 says that

$$
\left(d^{4}, e^{4}\right) A_{\mathfrak{m}}: c^{4} \subseteq\left(d^{4}, e^{4}\right) A_{\mathfrak{m}}: \mathfrak{m}^{2} A_{\mathfrak{m}}
$$

and hence $\left(d^{4}, e^{4}\right) A: c^{4} /\left(d^{4}, e^{4}\right) A$ has finite length. Therefore we obtain an exact sequence

$$
\begin{aligned}
0 \rightarrow & \frac{\left(d^{4}, e^{4}\right) A: c^{4}}{\left(d^{4}, e^{4}\right)}(-4) \rightarrow H_{\mathfrak{m}}^{0}\left(A /\left(d^{4}, e^{4}\right)\right)(-4) \\
& \stackrel{c^{4}}{\longrightarrow} H_{\mathfrak{m}}^{0}\left(A /\left(d^{4}, e^{4}\right)\right) \rightarrow H_{\mathfrak{m}}^{0}\left(A /\left(c^{4}, d^{4}, e^{4}\right)\right) \rightarrow H_{\mathfrak{m}}^{1}\left(A /\left(d^{4}, e^{4}\right)\right)(-4) \\
& \stackrel{c^{4}}{\longrightarrow} H_{\mathfrak{m}}^{1}\left(A /\left(d^{4}, e^{4}\right)\right) \rightarrow H_{\mathfrak{m}}^{1}\left(A /\left(c^{4}, d^{4}, e^{4}\right)\right) \rightarrow H_{\mathfrak{m}}^{2}\left(A /\left(d^{4}, e^{4}\right)\right)(-4) \\
& \stackrel{c^{4}}{\longrightarrow} H_{\mathfrak{m}}^{2}\left(A /\left(d^{4}, e^{4}\right)\right) \rightarrow \cdots
\end{aligned}
$$

See [11 Proposition 2.6]. By comparing degree, we have two split short exact sequences

$$
\begin{aligned}
0 & \rightarrow H_{\mathfrak{m}}^{0}\left(A /\left(d^{4}, e^{4}\right)\right) \rightarrow H_{\mathfrak{m}}^{0}\left(A /\left(c^{4}, d^{4}, e^{4}\right)\right) \rightarrow H_{\mathfrak{m}}^{1}\left(A /\left(d^{4}, e^{4}\right)\right)(-4) \rightarrow 0 \\
0 & \rightarrow H_{\mathfrak{m}}^{1}\left(A /\left(d^{4}, e^{4}\right)\right) \rightarrow H_{\mathfrak{m}}^{1}\left(A /\left(c^{4}, d^{4}, e^{4}\right)\right) \rightarrow H_{\mathfrak{m}}^{2}\left(A /\left(d^{4}, e^{4}\right)\right)(-4) \rightarrow 0 .
\end{aligned}
$$

Therefore

$$
\begin{aligned}
& H_{\mathfrak{m}}^{0}\left(A /\left(c^{4}, d^{4}, e^{4}\right)\right)=(A / \mathfrak{m})^{3}(-6), \\
& H_{\mathfrak{m}}^{1}\left(A /\left(c^{4}, d^{4}, e^{4}\right)\right)=A / \mathfrak{m}(-2)
\end{aligned}
$$

and hence $\mathfrak{a}\left(A_{\mathfrak{m}} /\left(c^{4}, d^{4}, e^{4}\right)\right)=\mathfrak{m}^{2} A_{\mathfrak{m}} \ni b^{4}$.

Similarly we have $H_{\mathfrak{m}}^{0}\left(A /\left(b^{4}, c^{4}, d^{4}, e^{4}\right)\right)=(A / \mathfrak{m})^{4}(-6)$ and

$$
\mathfrak{a}\left(A_{\mathfrak{m}} /\left(b^{4}, c^{4}, d^{4}, e^{4}\right) A_{\mathfrak{m}}\right)=\mathfrak{m} A_{\mathfrak{m}} \ni a^{4} .
$$

Although the finite morphism

$$
\operatorname{Spec} k[a, b, c, d, e]_{(a, b, c, d, e)} \rightarrow \operatorname{Spec} A_{\mathfrak{m}}
$$

is a Macaulayfication, we construct a Macaulayfication of Spec $A_{\mathfrak{m}}$ by using Corollary 4.2. Let $\mathfrak{b}=\prod_{i=1}^{4}\left(x_{i}, \ldots, x_{5}\right) A_{\mathfrak{m}}$ and $Y=\operatorname{Proj} R(\mathfrak{b})$. From the proof of 
Lemma 4.3, we find that $Y$ is covered by 16 affine schemes:
(1) $\quad \operatorname{Spec} A_{\mathfrak{m}}\left[\frac{a^{4}}{e^{4}}, \frac{b^{4}}{e^{4}}, \frac{c^{4}}{e^{4}}, \frac{d^{4}}{e^{4}}\right]$,
(2) $\operatorname{Spec} A_{\mathfrak{m}}\left[\frac{e^{4}}{a^{4}}, \frac{b^{4}}{e^{4}}, \frac{c^{4}}{e^{4}}, \frac{d^{4}}{e^{4}}\right]$,
(3) $\operatorname{Spec} A_{\mathfrak{m}}\left[\frac{a^{4}}{b^{4}}, \frac{e^{4}}{b^{4}}, \frac{c^{4}}{e^{4}}, \frac{d^{4}}{e^{4}}\right]$,
(4) $\operatorname{Spec} A_{\mathfrak{m}}\left[\frac{b^{4}}{a^{4}}, \frac{e^{4}}{b^{4}}, \frac{c^{4}}{e^{4}}, \frac{d^{4}}{e^{4}}\right]$,
(5) $\quad \operatorname{Spec} A_{\mathfrak{m}}\left[\frac{a^{4}}{c^{4}}, \frac{b^{4}}{c^{4}}, \frac{e^{4}}{c^{4}}, \frac{d^{4}}{e^{4}}\right]$,
(6) Spec $A_{\mathfrak{m}}\left[\frac{c^{4}}{a^{4}}, \frac{b^{4}}{c^{4}}, \frac{e^{4}}{c^{4}}, \frac{d^{4}}{e^{4}}\right]$,
(7) $\operatorname{Spec} A_{\mathfrak{m}}\left[\frac{a^{4}}{b^{4}}, \frac{c^{4}}{b^{4}}, \frac{e^{4}}{c^{4}}, \frac{d^{4}}{e^{4}}\right]$,
(8) $\operatorname{Spec} A_{\mathfrak{m}}\left[\frac{b^{4}}{a^{4}}, \frac{c^{4}}{b^{4}}, \frac{e^{4}}{c^{4}}, \frac{d^{4}}{e^{4}}\right]$,
(9) $\quad \operatorname{Spec} A_{\mathfrak{m}}\left[\frac{a^{4}}{d^{4}}, \frac{b^{4}}{d^{4}}, \frac{c^{4}}{d^{4}}, \frac{e^{4}}{d^{4}}\right]$,
(10) $\operatorname{Spec} A_{\mathfrak{m}}\left[\frac{d^{4}}{a^{4}}, \frac{b^{4}}{d^{4}}, \frac{c^{4}}{d^{4}}, \frac{e^{4}}{d^{4}}\right]$,
(11) $\operatorname{Spec} A_{\mathfrak{m}}\left[\frac{a^{4}}{b^{4}}, \frac{d^{4}}{b^{4}}, \frac{c^{4}}{d^{4}}, \frac{e^{4}}{d^{4}}\right]$,
(12) Spec $A_{\mathfrak{m}}\left[\frac{b^{4}}{a^{4}}, \frac{d^{4}}{b^{4}}, \frac{c^{4}}{d^{4}}, \frac{e^{4}}{d^{4}}\right]$,
(13) Spec $A_{\mathfrak{m}}\left[\frac{a^{4}}{c^{4}}, \frac{b^{4}}{c^{4}}, \frac{d^{4}}{c^{4}}, \frac{e^{4}}{d^{4}}\right]$,
(14) $\operatorname{Spec} A_{\mathfrak{m}}\left[\frac{c^{4}}{a^{4}}, \frac{b^{4}}{c^{4}}, \frac{d^{4}}{c^{4}}, \frac{e^{4}}{d^{4}}\right]$,
(15) $\quad$ Spec $A_{\mathfrak{m}}\left[\frac{a^{4}}{b^{4}}, \frac{c^{4}}{b^{4}}, \frac{d^{4}}{c^{4}}, \frac{e^{4}}{d^{4}}\right]$,
(16) $\quad \operatorname{Spec} A_{\mathfrak{m}}\left[\frac{b^{4}}{a^{4}}, \frac{c^{4}}{b^{4}}, \frac{d^{4}}{c^{4}}, \frac{e^{4}}{d^{4}}\right]$.

We check the Cohen-Macaulay property of them.

For example, we consider (16). By letting $s=a, t=b / a, u=c / b, v=d / c$ and $w=e / d$, we obtain an isomorphism

$$
\begin{gathered}
A\left[b^{4} / a^{4}, c^{4} / b^{4}, d^{4} / c^{4}, e^{4} / d^{4}\right] \\
\cong k\left[s, t^{4}, u^{4}, v^{4}, w^{4}, s t, s t u, s t u v, s^{2} t^{2} u^{2} v^{2} w^{2}, s^{3} t^{3} u^{3} v^{3} w^{3},\right. \\
\left.\quad s^{3} t^{2} u^{2} v^{2} w, s^{3} t^{3} u^{2} v^{2} w, s^{3} t^{3} u^{3} v^{2} w, s^{3} t^{3} u^{3} v^{3} w\right] .
\end{gathered}
$$

The right hand side is also an affine semigroup ring and satisfies the assumption of the following theorem.

Theorem B.2 ([13, Theorem 2.6]). Let $k$ be a field and $B=k\left[m_{1}, \ldots, m_{r}\right]$ an affine semigroup ring, where $m_{1}, \ldots, m_{r}$ are monomials. Assume that

1. $m_{1}, \ldots, m_{d}$ are algebraically independent over $k$;

2. there is a positive integer $k$ such that $m_{d+1}^{k}, \ldots, m_{r}^{k} \in k\left[m_{1}, \ldots, m_{d}\right]$.

Then the following statements are equivalent:

1. $B$ is Cohen-Macaulay;

2. $m_{i} B: m_{j}=m_{i} B$ for all $i<j$.

It is easy to see that $A\left[b^{4} / a^{4}, c^{4} / b^{4}, d^{4} / c^{4}, e^{4} / d^{4}\right]$ is a Cohen-Macaulay ring by using the theorem above and we can prove that (1)-(15) are Cohen-Macaulay in the same way.

\section{REFERENCES}

[1] Yoichi Aoyama and Shiro Goto, Some special cases of a conjecture of Sharp, J. Math. Kyoto Univ. 26 (1986), 613-634. MR 88h:13013

[2] — A conjecture of Sharp - the case of local rings with $\operatorname{dim}$ non-CM $\leq 1$ or $\operatorname{dim} \leq 5$, Algebraic Geometry and Commutative Algebra in Honor of Masayoshi Nagata, Kinokuniya, 1988, pp. 27-34. MR 90b:13018 
[3] Dave Bayer and Michael Stillman, Macaulay: A system for computation in algebraic geometry and commutative algebra, 1982-1994, Source and object code available for Unix and Macintosh computers. Contact the authors, or download from math.harvard.edu via anonymous ftp.

[4] Markus Brodmann, Kohomologische Eigenschaften on Audblasungen an Lokal Vollständgen Durchschnitten, 1980, Habilitationsschrift.

[5] _ Two types of birational models, Comment. Math. Helv. 58 (1983), 388-415. MR 85g:14061

[6] - A few remarks on blowing-up and connectedness, J. Reine Ang199ew. Math. 370 (1986), 52-60. MR 87j:14014

[7] Nguyen Tu Cuong, P-standard systems of parameters and p-standard ideals in local rings, Acta Math. Vietnamica 20 (1995), 145-161.

[8] Gerd Faltings, Über die Annulatoren lokaler Kohomogiegruppen, Arch. Math. (Basel) 30 (1978), 473-476. |MR 58:22058

[9] _ Über Macaualyfizierung, Math. Ann. 238 (1978), 175-192. MR 80c:14030

[10] Shiro Goto, On the Cohen-Macaulayfication of certain Buchsbaum rings, Nagoya Math. J. 80 (1980), 107-116. MR 81m:13023

[11] _ Blowing-up of Buchsbaum rings, Proceedings, Durham symposium on Commutative Algebra, London Math. Soc. Lect. Notes, vol. 72, Cambridge Univ. Press, 1982, pp. 140-162. MR 84h:13032

[12] Shiro Goto and Yasuhiro Shimoda, On Rees algebras over Buchsbaum rings, J. Math. Kyoto Univ. 20 (1980), 691-708. MR 82c: 13027

[13] Shiro Goto, Naoyoshi Suzuki, and Kei-ichi Watanabe, On affine semigroup rings, Japan. J. Math. (N.S.) 2 (1976), 1-12. MR 56:8553

[14] Shiro Goto and Kei-ichi Watanabe, On graded rings, I, J. Math. Soc. Japan 30 (1978), 179-213. MR 81m:13021

[15] Shiro Goto and Kikumichi Yamagishi, The theory of unconditioned strong d-sequences and modules of finite local cohomology, preprint.

[16] A. Grothendieck, Éléments de Géométrie Algébrique II, Inst. Hautes Études Sci. Publ. Math., vol. 8, Presses Universitaires de France, 1961. MR 29:1208

[17] Robin Hartshorne, Residue and duality, Lecture Notes in Math., vol. 20, Springer-Verlag, Berlin, Heidelberg, New York, 1966. MR 36:5145

[18] — Algebraic geometry, Graduate Texts in Mathematics, vol. 52, Springer, Berlin, Heidelberg, New-York, 1977. MR 57:3116

[19] Heisuke Hironaka, Resolution of singularities of an algebraic variety over a field of characteristic 0, Ann. of Math. (2) 79 (1964), 109-326. MR 33:7333

[20] Craig Huneke, The theory of d-sequences and powers of ideals, Adv. in Math. 46 (1982), 249-279. MR 84g:13021

[21] Takesi Kawasaki, On Macaulayfication of certain quasi-projective schemes, J. Math. Soc. Japan 50 (1998), 969-991. CMP 99:01

[22] Hideyuki Matsumura, Commutative ring theory, Cambridge Studies in Advanced Math., vol. 8, Cambridge University Press, 1986. MR 90i:13001

[23] D. G. Northcott and D. Rees, Reductions of ideals in local rings, Proc. Cambridge. Phil. Soc. 50 (1954), 145-158. MR 15:596a

[24] Tetsushi Ogoma, Existence of dualizing complexes, J. Math. Kyoto Univ. 24 (1984), 27-48. MR 85j:13028

[25] Idun Reiten, The converse to a theorem of Sharp in Gorenstein modules, Proc. Amer. Math. Soc. 32 (1972), 417-420. MR 45:5128

[26] Peter Schenzel, Dualizing complexes and system of parameters, J. Algebra 58 (1979), 495501. MR 80f:14025

[27] Cohomological annihilators, Math. Proc. Cambridge Philos. Soc. 91 (1982), 345-350. MR 83j:13008

[28] Dualisierende Komplexe in der lokalen Algebra und Buchsbaum ringe, Lecture Notes in Math., vol. 907, Springer, Berlin, Heidelberg, New York, 1982. MR 83i:13013

[29] _ Standard system of parameters and their blowing-up rings, J. Reine Angew. Math. 344 (1983), 201-220. MR 84m:13025 
[30] Rodney Y. Sharp, Necessary conditions for the existence of dualizing complexes in commutative algebra, Sém. Algèbre P. Dubreil 1977/78, Lecture Notes in Mathematics, vol. 740, Springer-Verlag, 1979, pp. 213-229. MR 81d:13013

[31] Giuseppe Valla, Certain graded algebras are always Cohen-Macaulay, J. Algebra 42 (1976), 537-548. MR 54:10240

Department of Mathematics, Tokyo Metropolitan University, Hachioji-shi MinamiOHSAWA 1-1, TOKYo 192-0397, JAPAN

E-mail address: kawasaki@comp.metro-u.ac.jp 